\title{
Supersymmetry Breaking in Low Dimensional Models
}

\author{
Christian Wozar, Andreas Wipf \\ Theoretisch-Physikalisches Institut, Universität Jena, D-07743 Jena, Germany
}

\begin{abstract}
We analyse supersymmetric models that show supersymmetry breaking in one and two dimensions using lattice methods. Starting from supersymmetric quantum mechanics we explain the fundamental principles and problems that arise in putting supersymmetric models onto the lattice. We compare our lattice results (built upon the non-local SLAC derivative) with numerically exact results obtained within the Hamiltonian approach. A particular emphasis is put on the discussion of boundary conditions. We investigate the ground state structure, mass spectrum, effective potential and Ward identities and conclude that lattice methods are suitable to derive the physical properties of supersymmetric quantum mechanics, even with broken supersymmetry. Based on this result we analyse the two dimensional $\mathcal{N}=1$ Wess-Zumino model with spontaneous supersymmetry breaking. First we show that (in agreement with earlier analytical and numerical studies) the SLAC derivative is a sensible choice in the quenched model, which is nothing but the two dimensional $\phi^{4}$ model. Then, we present the very first computation of a renormalised critical coupling for the complete supersymmetric model. This calculation makes use of Binder cumulants and is supported by a direct comparison to Ward identity results, both in the continuum and infinite volume limit. The physical picture is completed by masses at two selected couplings, one in the supersymmetric phase and one in the supersymmetry broken phase. Signatures of the Goldstino in the fermionic correlator are clearly visible in the broken case.
\end{abstract}

Keywords: Supersymmetric quantum mechanics, Wess-Zumino model, Lattice models, Supersymmetry breaking PACS: 12.60.Jv, 11.30.Pb, 11.15.Ha, 11.10.Ef, 11.10.Gh

\section{Introduction}

Symmetries are one of the guiding principles in contemporary theoretical physics. They led to the construction of the standard model of electroweak interaction [1-3] and strong interaction [4]. Based on the underlying symmetries bottom and top quark as well as the $\tau$ neutrino have been predicted and the experimental discoveries, the last one more than two decades after the prediction [5], substantiated the success of the standard model to describe the physics on energy scales below $1 \mathrm{TeV}$. The extension of the standard model's symmetries by further ones is constrained by the celebrated Coleman-Mandula theorem [6]. A way to circumvent this no-go theorem is given by extending the Poincaré algebra with anti-commuting supersymmetry generators [7] that relate particles with integer spin to ones with half-integer spin.

The first field theoretical realisation of a renormalisable model with supersymmetry is the Wess-Zumino model [8] in four spacetime dimensions with a field content of two (real) scalars and a Majorana fermion. Since then a variety of models with global supersymmetry have been constructed, for example supersymmetric gauge theories or supersymmetric sigma models and some of these possess several supersymmetries. For an introduction, see [9-11]. Supersymmetric extensions of the well established standard model may solve or weaken several problems of the standard model, such as the hierarchy problem, the occurrence of dark matter and the strong CP problem [12-14].

Supersymmetric models have certain theoretical advantages, such as less severe divergences in perturbation theory as compared to models without supersymmetry, and the supersymmetry algebra induces a vanishing ground state energy, as long as supersymmetry is unbroken. If the ground state is invariant under supersymmetry, mass degenerate

Email addresses: christian.wozar@uni-jena.de (Christian Wozar), wipf@tpi.uni-jena.de (Andreas Wipf) 
multiplets of bosonic and fermionic particles are predicted, and it known that in certain classes of supersymmetric theories a spontaneous breaking of supersymmetry is not possible [15]. In experiments no such mass degeneracy has been detected so far, and the masses of bosonic and fermionic particles appear to be unrelated. At first sight these results tell us that supersymmetry is not realised in nature. However, as first analysed by O'Raifeartaigh [16] this non-degeneracy of masses is naturally expected if supersymmetry is dynamically broken.

In a theory with dynamical supersymmetry breaking the ground state is not invariant under supersymmetry, and the ground state energy is lifted above zero [17]. But the supersymmetry algebra is still present and this has implications for the physics in the dynamically broken sector. To date the Large Hadron Collider is fully operating and it is expected to measure remnants of supersymmetry in collision events within the upcoming years. Clearly, if supersymmetry plays any role in nature then it is mandatory to explore supersymmetric theories with methods that are applicable in the nonperturbative regime.

Among those methods the lattice regularisation in combination with importance sampling based statistical 'MonteCarlo' methods has been most successful over the last decades. Lattice methods often provide the only viable way to gain information about the non-perturbative sector of quantum field theories. Early simulations that aimed at an understanding of the pure $S U(2)$ Yang-Mills theory [18] built the basis for recent computations from first principles of the Hadron spectrum in full quantum chromodynamics [19], which is only possible due to increasing computing power and algorithmic improvements. As non-perturbative effects are automatically taken into account in lattice simulations, it is desirable to apply the lattice approach to supersymmetric theories as well. This has been the subject of a number of publications, see, e.g., [20-24] and for recent progress in supersymmetric Yang-Mills theories [25-28] and references therein.

In all lattice regularised versions of field theories symmetries are of particular interest. If a symmetry of the continuum theory is not implemented in the lattice version it may happen that the symmetry is not restored in the continuum limit. E.g. for simulations of gauge theories it is important to implement the lattice version of the continuum gauge symmetry [29]. But not every symmetry can be directly implemented in the lattice regularised theory. For instance, the Nielsen-Ninomiya theorem [30-32] forbids the exact implementation of chirally symmetric fermions with a local fermion interaction and without introducing additional fermion flavours on the lattice. Nevertheless, it is possible to construct a (deformed) lattice version of the chiral symmetry, which is given by the Ginsparg-Wilson relation [33], so that a restoration of the continuum chiral symmetry is ensured in the continuum limit of the lattice action.

For supersymmetry as extension of the Poincaré algebra a fully realised supersymmetry algebra on the lattice must inevitably contain the generators of translations which would imply arbitrary translations to be part of the symmetry group of the lattice theory. By contrast, lattice regularised theories are only symmetric under translations by the lattice spacing. Therefore a complete realisation of the continuum supersymmetry algebra on the lattice is impossible and the full supersymmetry can only be realised as an accidental symmetry in the continuum limit of the lattice regularised theory. Technically, the reason for this can be traced back to the failure of the Leibniz rule on the lattice [34].

It has been shown that even in supersymmetric quantum mechanics a naive discretisation does not lead to a supersymmetric continuum limit [35]; generically, such a limit can at best be achieved by fine-tuning the bare coefficients of all supersymmetry-breaking counterterms [36]. This, however, requires much knowledge of the theory in advance. In some cases the relevant operators can be determined perturbatively, cf. [37]. To circumvent the fine-tuning process several approaches are conceivable. Firstly a partial realisation of supersymmetry on the lattice is possible for theories with extended supersymmetry (for a review see [38]). Secondly recent developments aim at the construction of a Ginsparg-Wilson inspired relation for supersymmetric theories to obtain a lattice version of supersymmetry such that the continuum supersymmetry is broken in a controlled way [39]. Alternatively for scalar theories a deformed supersymmetry algebra on the lattice can be constructed by using a non-local product such that the theory is invariant under the full (deformed) lattice supersymmetry [40, 41].

Apart from an explicit supersymmetry breaking by the finite lattice spacing there exist further supersymmetry breaking effects that must be controlled in the analysis of supersymmetric theories. For example, at finite temperature Lorentz invariance and therefore supersymmetry as extension of the Poincaré symmetry are broken 1 In addition, for a finite spatial volume there may exist tunnelling processes between two formerly separate ground states such

\footnotetext{
${ }^{1}$ This problem can be avoided by choosing periodic boundary conditions also for the fermions. However, this is only possible for an unbroken supersymmetry.
} 
that the finite volume ground state energy is raised above zero. As it is inevitably to use finite lattices for numerical simulations these explicit supersymmetry breaking effects must be taken into account.

We begin our investigations with discretised supersymmetric quantum mechanics with dynamically broken supersymmetry in Sec. 2. In this setting the basic concepts of supersymmetric theories are explained and reference results for certain observables are computed via the operator formalism, thus allowing to understand the physics behind supersymmetry breaking on solid grounds. The corresponding lattice regularisation is based on a formulation that has been used in the unbroken supersymmetric quantum mechanics with great success [42]. We verify that even for quantum mechanical models with broken supersymmetry it is possible to obtain accurate results on the low lying energy spectrum from lattice simulations.

The minimal setting for a field theory with supersymmetry breaking phase transition is given by the $\mathcal{N}=1$ WessZumino model in $1+1$ dimensions, which is analysed in Sec. 3 In the context of the quenched model a particular renormalised critical coupling for the $\mathbb{Z}_{2}$ symmetry breaking is shown to be independent of the chosen lattice regulator. The corresponding critical coupling in the full theory is determined and the relation between $\mathbb{Z}_{2}$ and supersymmetry breaking is worked out.

\section{Broken supersymmetric quantum mechanics}

An extensive analysis of quantum mechanical systems, such as the anharmonic oscillator, with lattice regularised path integrals has been performed almost three decades ago [43]. There has been renewed interest in quantum mechanical systems on the lattice in the context of supersymmetric quantum mechanics (SQM). In several works SQM has been used as a toy model to study the supersymmetry breaking induced by a naive lattice formulation [44] and to explore lattice regularisations with partially [35, 42, 45, 46] or fully [40] conserved supersymmetries. It has been pointed out that a discretisation without any conserved supersymmetries may not be free of finite supersymmetry breaking renormalisation terms in the continuum limit [47] such that a careful treatment of supersymmetry restoration is needed. Most of the lattice studies of SQM so far have been carried out for the case of an unbroken supersymmetry and only few of them [48, 49] consider the case of the dynamically broken supersymmetry. Here, the case of a SQM with dynamically broken supersymmetry is considered to explain the concepts and effects of supersymmetry breaking in a setting that allows for high precision measurements in the lattice theory and provides the possibility to compare to exactly calculable reference values from the operator formalism.

\subsection{Operator formalism}

SQM in one dimension is a generalisation of the supersymmetric harmonic oscillator 2 In analogy to supersymmetric field theories nilpotent supercharges $\mathcal{Q}$ and its adjoint $\mathcal{Q}^{\dagger}$ are introduced,

$$
\mathcal{Q}^{\dagger}=\left(\begin{array}{ll}
0 & 0 \\
A & 0
\end{array}\right)=A \Psi^{\dagger}, \quad \mathcal{Q}=\left(\begin{array}{cc}
0 & A^{\dagger} \\
0 & 0
\end{array}\right)=A^{\dagger} \Psi
$$

with fermionic creation and annihilation operator $\Psi^{\dagger}$ and $\Psi$ and first order differential operators

$$
A=\frac{\mathrm{d}}{\mathrm{d} \phi}+P(\phi), \quad A^{\dagger}=-\frac{\mathrm{d}}{\mathrm{d} \phi}+P(\phi)
$$

containing the prepotential $P(\phi)$. In accordance to the field theory language we denote the position operator of the quantum mechanical system by $\phi$. The Hamiltonian is constructed via

$$
\frac{1}{2}\left\{\mathcal{Q}, \mathcal{Q}^{\dagger}\right\}=\left(\begin{array}{cc}
H_{\mathrm{B}} & 0 \\
0 & H_{\mathrm{F}}
\end{array}\right)=\frac{1}{2}\left(-\frac{\mathrm{d}^{2}}{\mathrm{~d} \phi^{2}}+P^{2}(\phi)+\left[\Psi^{\dagger}, \Psi\right] P^{\prime}(\phi)\right) \equiv H,
$$

and acts on two-component state vectors $|\psi\rangle=\left(|\psi\rangle_{\mathrm{B}},|\psi\rangle_{\mathrm{F}}\right)^{\top}$ where, for convenience, the first component is called 'bosonic' and the second one 'fermionic'. The supersymmetry algebra is completed by the nilpotency of $\mathcal{Q}$ and $\mathcal{Q}^{\dagger}$ and the commutation with $H$,

$$
\{\mathcal{Q}, \mathcal{Q}\}=0, \quad\left\{\mathcal{Q}^{\dagger}, \mathcal{Q}^{\dagger}\right\}=0, \quad[\mathcal{Q}, H]=0 .
$$

\footnotetext{
${ }^{2}$ An extended introduction to the operator formalism for supersymmetric quantum mechanics can be found in [50, 51].
} 


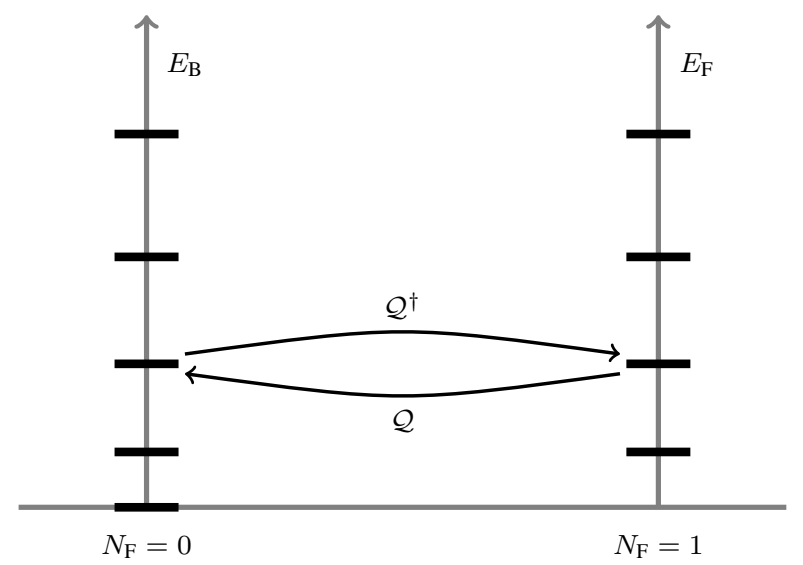

Figure 1: Energy spectrum for the unbroken supersymmetric quantum mechanics discussed in [42]. $\mathcal{Q}$ and $\mathcal{Q}^{\dagger}$ map between bosonic and fermionic sector.

If $P$ is a linear function of $\phi$ then $A$ and $A^{\dagger}$ are the bosonic annihilation and creation operators of the (supersymmetric) harmonic oscillator. Accordingly the bosonic and fermionic Hamiltonian is given by

$$
H_{\mathrm{B}}=\frac{1}{2} A^{\dagger} A=-\frac{1}{2} \frac{\mathrm{d}^{2}}{\mathrm{~d} \phi^{2}}+V_{\mathrm{B}}, \quad H_{\mathrm{F}}=\frac{1}{2} A A^{\dagger}=-\frac{1}{2} \frac{\mathrm{d}^{2}}{\mathrm{~d} \phi^{2}}+V_{\mathrm{F}}, \quad V_{\mathrm{B} / \mathrm{F}}=\frac{1}{2}\left(P^{2}(\phi) \mp P^{\prime}(\phi)\right) .
$$

Both Hamiltonians are by construction non-negative. The bosonic sector of a zero energy state is annihilated by $A$ and a fermionic one is annihilated by $A^{\dagger}$,

$$
H_{\mathrm{B}}|0\rangle_{\mathrm{B}}=0 \Leftrightarrow A|0\rangle_{\mathrm{B}}=0, \quad H_{\mathrm{F}}|0\rangle_{\mathrm{F}}=0 \Leftrightarrow A^{\dagger}|0\rangle_{\mathrm{F}}=0
$$

The supersymmetry algebra implies a strict pairing of excited states, i.e. for every bosonic eigenstate $\left|\psi_{\mathrm{B}}\right\rangle$ with energy $E>0$ there is a fermionic partner state

$$
\left|\psi_{\mathrm{F}}\right\rangle=\frac{1}{\sqrt{2 E}} \mathcal{Q}^{\dagger}\left|\psi_{\mathrm{B}}\right\rangle, \quad\left|\psi_{\mathrm{B}}\right\rangle=\frac{1}{\sqrt{2 E}} \mathcal{Q}\left|\psi_{\mathrm{F}}\right\rangle
$$

with identical norm and energy.

The zero energy state(s) of the super Hamiltonian $H$ can be given explicitly (in position space) as solutions of first order differential equations. If one of these functions is normalisable, then the supersymmetric ground state exists and supersymmetry is unbroken. Since the product of possible zero energy states $\left\langle x \mid 0_{\mathrm{B}}\right\rangle \cdot\left\langle x \mid 0_{\mathrm{F}}\right\rangle$ is constant, there is at most one normalisable state with zero energy. The explicit form of solutions implies that for a polynomial prepotential $P(\phi)=\sum_{n=0}^{N} c_{n} \phi^{n}$ with $c_{N} \neq 0$ supersymmetry is unbroken iff $N$ is odd. In that case there is one normalisable zero energy state and the spectrum is similar to the one depicted in Fig. 1.

\subsubsection{Supersymmetry breaking and the Witten index}

An existing and unbroken supersymmetry is defined by the existence of a normalisable ground state $|0\rangle$ which is annihilated by $\mathcal{Q}$ and $\mathcal{Q}^{\dagger}$ which implies $H_{\mathrm{B}}|0\rangle_{\mathrm{B}}=H_{\mathrm{F}}|0\rangle_{\mathrm{F}}=0$. Witten introduced an index [15] to determine whether supersymmetry can be broken dynamically in supersymmetric field theories. In the present context it is given by the trace over all eigenstates of $H$,

$$
\Delta=\operatorname{Tr}(-1)^{N_{\mathrm{F}}},
$$

where $N_{\mathrm{F}}=\left(\begin{array}{ll}0 & 0 \\ 0 & 1\end{array}\right)$ is the fermion number operator that commutes with $H 3^{3}$ Now, two alternatives exist:

\footnotetext{
${ }^{3}$ As it stands, $\Delta$ is not well defined and requires a normalisation, e.g. $\Delta=\lim _{\beta \rightarrow 0} \operatorname{Tr}\left[\mathrm{e}^{-\beta H}(-1)^{N_{\mathrm{F}}}\right]$.
} 


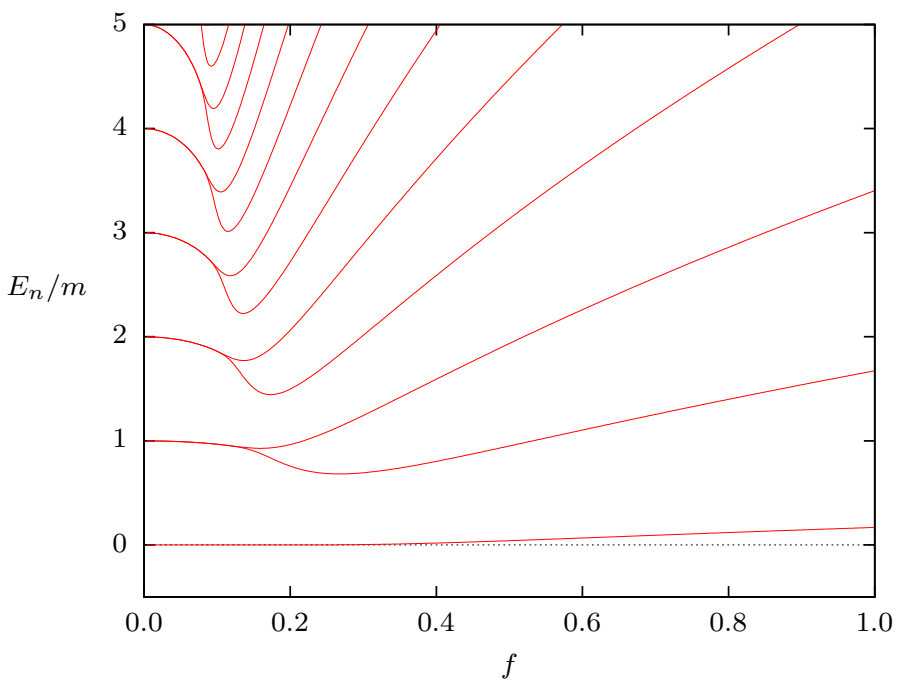

Figure 2: Energy levels for the broken supersymmetric quantum mechanics. Each level is doubly degenerate with one bosonic and one fermionic eigenstate.

- For broken supersymmetry there is no normalisable zero energy state. All eigenstates of $H$ have positive energies and must be paired, which implies $\Delta=0$.

- For unbroken supersymmetry there are $n_{\mathrm{B}}$ bosonic and $n_{\mathrm{F}}$ fermionic ground states with zero energy. They contribute with $n_{\mathrm{B}}-n_{\mathrm{F}}$ to the Witten index. All contributions from the excited states cancel, which gives $\Delta=n_{\mathrm{B}}-n_{\mathrm{F}}$.

Therefore a non-vanishing Witten index implies an unbroken supersymmetry, but not necessarily vice versa. It is still possible that supersymmetry is unbroken while there are the same number of bosonic and fermionic zero energy states. For a one dimensional supersymmetric quantum mechanics at most one zero energy state is possible and $\Delta \neq 0$ is equivalent to unbroken supersymmetry.

\subsubsection{Specifying the model}

The minimal modification of the supersymmetric harmonic oscillator with broken supersymmetry is given by the prepotential

$$
P(\phi)=m \phi+h \phi^{2}
$$

with vanishing Witten index. Hence there is no normalisable ground state with zero energy. The spectrum is completely degenerate and acting with the supercharges on one finite energy ground state will give the corresponding superpartner of this ground state 4

This model depends on the dimensionful parameters $m$ and $h$ and in analogy to the supersymmetric harmonic oscillator $m$ is used to set the scale. Therefore $f=h / m^{1.5}$ provides a scale independent dimensionless coupling. In a heat bath the dimensionless temperature is given by $T=(m \beta)^{-1}$ with $\beta$ as dimensionful inverse temperature. Eventually coordinates are made dimensionless by setting $\Phi=\phi \sqrt{m}$.

The energy spectrum and corresponding states can be computed directly by a discretisation of position space and replacement of $\frac{\mathrm{d}}{\mathrm{d} \phi}$ by a discretised derivative. After the analysis of different possible discretisations in [52] the numerically most stable choice is the SLAC derivative [53], which for periodic boundary conditions on a lattice with

\footnotetext{
${ }^{4}$ If not otherwise stated, "ground states" may also have a positive energy.
} 


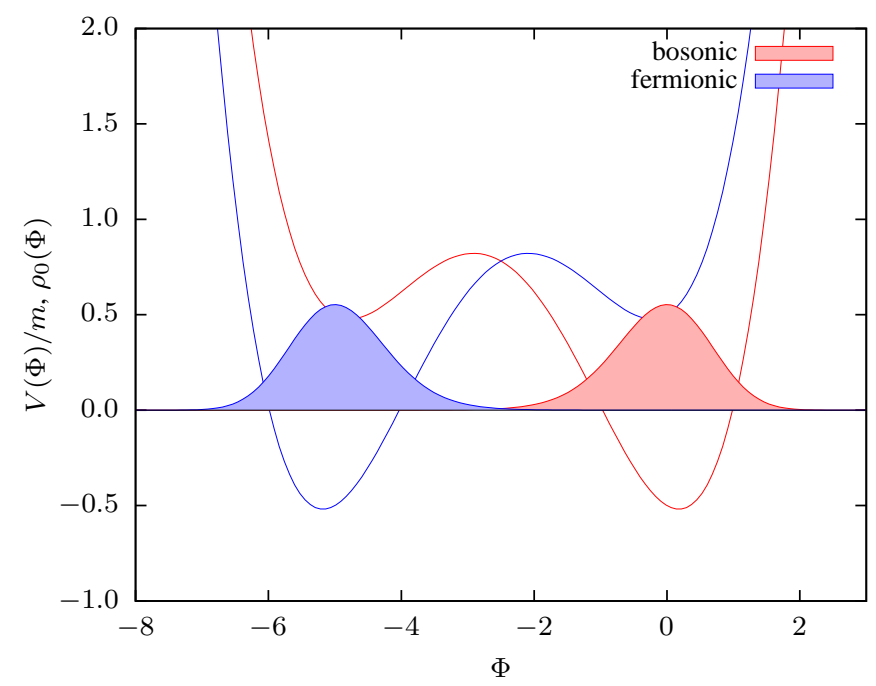

Figure 3: Probability density $\rho_{0}(\Phi)$ (shaded areas) for bosonic and fermionic ground state and potentials $V_{\mathrm{B} / \mathrm{F}}$ (lines) of the corresponding Hamiltonian at coupling $f=0.2$.

an odd number of points $N$ and lattice spacing $a$ is given by

$$
\begin{gathered}
a\left(\partial^{\mathrm{SLAC}}\right)_{x y}=\left\{\begin{array}{ll}
0 & : x=y \\
\frac{\pi}{N}(-)^{x-y} \frac{1}{\sin (\pi(x-y) /(N a))} & : x \neq y
\end{array},\right. \\
-a^{2}\left(\partial^{\mathrm{SLAC}}\right)_{x y}^{2}=\left\{\begin{array}{ll}
\frac{\pi^{2}}{N^{2}} \frac{N^{2}-1}{3} & : x=y \\
\frac{2 \pi^{2}}{N^{2}}(-)^{x-y} \frac{\cos (\pi(x-y) /(N a))}{\sin ^{2}(\pi(x-y) /(N a))} & : x \neq y
\end{array} .\right.
\end{gathered}
$$

The spectrum of the diagonalised Hamiltonian is depicted in Fig. 2 . For weak couplings $f \lesssim 0.1$ there is an additional (approximate) degeneracy of the excited spectrum corresponding to the perturbed energy levels of two harmonic oscillators with energies $\mathbb{N} m$ residing at the minima of the bosonic and fermionic potential $V_{\mathrm{B} / \mathrm{F}}$ (see Fig. 3).

Interpretation as a physical system. The naming 'bosonic' and 'fermionic' sector may sound misleading because of the complete degeneracy of the spectrum. The system can be interpreted as a particle with spin $1 / 2$ moving in an external potential that depends on the spin orientation. So 'bosonic' may refer to 'spin down' and 'fermionic' to 'spin up', respectively. Supersymmetry in this case is represented as degeneracy between an up and a down state. For the case of unbroken supersymmetry the ground state is unique and is invariant under application of supersymmetry although it is in a definite spin state given by the interaction potential. For the broken supersymmetry there are (in the present case) two different ground states none of which is energetically preferred (see Fig. 3). There is no interaction given by the Hamiltonian between bosonic and fermionic sector and one ground state will be preserved if no external interaction is applied (e.g. by interacting with a heat bath at finite temperature). Applying the supercharge will give the partner ground state and amounts to the symmetry between spin up and spin down state. Furthermore no linear combination of the two ground states is invariant under the supersymmetry.

Physics at $T=0$. At vanishing temperature physics is given by ground state (vacuum) expectation values. Since supersymmetry is broken the system will stay in one of the degenerate ground states and expectation values are defined by this particular ground state 5 Without loss of generality results are given for the bosonic (finite energy) ground state $\left|0_{\mathrm{B}}\right\rangle$ and the expectation value of an observable $\mathcal{O}$ is thus given by $\langle\mathcal{O}\rangle_{0}=\left\langle 0_{\mathrm{B}}|\mathcal{O}| 0_{\mathrm{B}}\right\rangle$.

\footnotetext{
${ }^{5}$ This is similar to the $\mathbb{Z}_{2}$ symmetry in the Ising chain. There, at any finite temperature the symmetry is restored. Only for $T=0$ the system will take (and preserve) one of the possible "ground states".
} 
Observables can be computed from the diagonalised Hamiltonian to provide reference values for lattice computations. This is done in the next section. On the lattice the primary focus lies on one- and two-point functions and the probability density of the coordinate $\Phi$ given by $\rho_{0}(\Phi)=|\langle\Phi \mid 0\rangle|^{2}$. The one-point function is then given by $\langle\Phi\rangle_{0}=\int \mathrm{d} \Phi \rho_{0}(\Phi) \Phi$

The bosonic two-point function (in the bosonic ground state) is defined through the Euclidean time evolution,

$$
\langle\Phi(t) \Phi(0)\rangle_{0}=\left\langle 0_{\mathrm{B}}|\Phi(t) \Phi(0)| 0_{\mathrm{B}}\right\rangle=\left\langle 0_{\mathrm{B}}\left|\mathrm{e}^{t H} \Phi \mathrm{e}^{-t H} \Phi\right| 0_{\mathrm{B}}\right\rangle=\left\langle 0_{\mathrm{B}}\left|\Phi \mathrm{e}^{-t\left(H-E_{0}\right)} \Phi\right| 0_{\mathrm{B}}\right\rangle .
$$

Equivalently the fermionic correlation function is computed by

$$
\left\langle\Psi(t) \Psi^{\dagger}(0)\right\rangle_{0}=\left\langle 0_{\mathrm{B}}\left|\Psi \mathrm{e}^{-t\left(H-E_{0}\right)} \Psi^{\dagger}\right| 0_{\mathrm{B}}\right\rangle .
$$

In each case $t m$ defines the dimensionless 'time'.

The last quantity of interest is the effective potential which may be either defined by a Legendre transform of the Schwinger function 6 or more directly at vanishing temperature by

$$
V_{\text {eff }}\left(\Phi_{0}\right)=\min _{\langle\psi|\Phi| \psi\rangle=\Phi_{0}}\langle\psi|H| \psi\rangle
$$

Finite temperature physics. For any finite temperature there is a Boltzmann distribution with the same contribution of bosonic and fermionic partner states, including the lowest energy ground states. Again, with high precision calculations of the low lying spectrum provided by the diagonalised Hamiltonian it is possible to compute the thermal field distribution and expectation values

$$
\rho_{T}(\Phi)=Z^{-1} \sum_{E} \mathrm{e}^{-E / T}\left|\left\langle\Phi \mid \psi_{E}\right\rangle\right|^{2}, \quad\langle\mathcal{O}\rangle_{T}=Z^{-1} \sum_{E}\left\langle\psi_{E}\left|\mathrm{e}^{-\beta H} \mathcal{O}\right| \psi_{E}\right\rangle, \quad Z=\sum_{E} \mathrm{e}^{-E / T}
$$

where the sums run over all bosonic and fermionic states.

\subsection{Lattice regularised path integral}

With the methods given in the previous section it is possible to obtain exact results against which the path integral based calculations can be compared. Therefore the accuracy of the lattice simulation can be determined even when supersymmetry is broken. The corresponding Euclidean path integral is given by

$$
\mathcal{Z}=\int \mathcal{D} \phi \mathcal{D} \psi \mathcal{D} \bar{\psi} \mathrm{e}^{-S[\phi, \psi, \bar{\psi}]}
$$

with Euclidean action

$$
S=\int \mathrm{d} \tau\left(\frac{1}{2}(\partial \phi)^{2}+\frac{1}{2} P^{2}(\phi)+\bar{\psi}\left(\partial+P^{\prime}(\phi)\right) \psi\right) .
$$

Expectation values are computed via

$$
\langle A\rangle=\mathcal{Z}^{-1} \int \mathcal{D} \phi \mathcal{D} \psi \mathcal{D} \bar{\psi} A[\phi, \psi, \bar{\psi}] \mathrm{e}^{-S[\phi, \psi, \bar{\psi}]} .
$$

Supersymmetry appears as a symmetry of the action, where one transformation is given by

$$
\delta^{(1)} \phi=\bar{\varepsilon} \psi, \quad \delta^{(1)} \bar{\psi}=-\bar{\varepsilon}(\dot{\phi}+P(\phi)), \quad \delta^{(1)} \psi=0
$$

and a variation of the action gives $\delta^{(1)} S=\int \mathrm{d} \tau[\partial(\bar{\varepsilon} P \psi)]=0$. In the same way the action allows for a second supersymmetry transformation

$$
\delta^{(2)} \phi=\bar{\psi} \varepsilon, \quad \delta^{(2)} \bar{\psi}=0, \quad \delta^{(2)} \psi=(\dot{\phi}-P) \varepsilon
$$

\footnotetext{
${ }^{6}$ The Schwinger function is naturally defined in a path integral formulation.
} 
For the above supersymmetries to hold it is necessary that the fields vanish at infinity or that they are periodic in the Euclidean time. But for a thermal path integral at inverse temperature $\beta$ the fields obey the boundary conditions

$$
\phi(0)=\phi(\beta), \quad \psi(0)=-\psi(\beta), \quad \bar{\psi}(0)=-\bar{\psi}(\beta),
$$

i.e. the fermionic field is antiperiodic in time. Since the fields need not vanish anymore the variation of the action then reads

$$
\delta^{(1)} S=[\bar{\varepsilon} P \psi]_{\tau=0}^{\beta}=-2[\bar{\varepsilon} P \psi]_{\tau=0}
$$

which can be non-vanishing so that supersymmetry is broken by the finite temperature. In [42, 44] for an unbroken supersymmetric quantum mechanics periodic boundary conditions have been used to avoid such an explicit breaking. For temperature going to zero a change in boundary conditions is equivalent to an insertion of $(-1)^{N_{\mathrm{F}}}$ into the path integral,

$$
\mathcal{Z}_{\mathrm{p}}=\int \mathcal{D} \phi \mathcal{D} \psi_{\mathrm{p}} \mathcal{D} \bar{\psi}_{\mathrm{p}} \mathrm{e}^{-S[\phi, \psi, \bar{\psi}]}=\int \mathcal{D} \phi \mathcal{D} \psi_{\text {ap }} \mathcal{D} \bar{\psi}_{\mathrm{ap}}(-1)^{N_{\mathrm{F}}} \mathrm{e}^{-S[\phi, \psi, \bar{\psi}]}=\mathcal{Z}_{\mathrm{ap}} \Delta .
$$

Here, the periodic path integral is vanishing due to $\Delta=0$ for a broken supersymmetry. Thus, for a theory allowing supersymmetry breaking, periodic (supersymmetry preserving) boundary conditions cause a severe sign problem. This does not completely rule out the choice of these boundary conditions, as will be discussed on the case of the two dimensional $\mathcal{N}=1$ Wess-Zumino model in Sec. 3.2. but puts constraints on the range of applicability 7 To have a well defined (non-vanishing) path integral antiperiodic (thermal) boundary conditions for the fermionic fields are imposed.

For a construction of a lattice model the choice of the lattice regularised derivative is crucial 8 The canonical choice for scalar theories would be the forward (or equivalently backward) derivative. For derivatives appearing in the fermionic action a popular choice is given by Wilson's prescription [29]. Nevertheless, these simple discretisation rules are not applicable to supersymmetric theories as analysed in [42, 44, 47] for the case of an unbroken supersymmetric quantum mechanics. These results show the need for a more careful treatment of the discretisation of supersymmetric theories. In the comparative study of six different discretisations [42] the one based on the SLAC derivative provides results close to the continuum limit even at finite lattice spacing. For an odd number of lattice points with periodic boundary conditions the matrix representation is already given in Eq. (10). Antiperiodic boundary conditions (necessary for fermionic fields) are best realised on an even lattice with $N$ points,

$$
a\left(\partial^{\mathrm{SLAC}}\right)_{x y}=\left\{\begin{array}{ll}
0 & : x=y \\
\frac{\pi}{N}(-)^{(x-y) / a} \frac{1}{\sin (\pi(x-y) /(N a))} & : x \neq y
\end{array},\right.
$$

while the squared SLAC derivative for an even number of lattice points and periodic boundary conditions (as needed for the bosonic fields) reads

$$
-a^{2}\left(\partial^{\mathrm{SLAC}}\right)_{x y}^{2}=\left\{\begin{array}{ll}
\frac{\pi^{2}}{N^{2}} \frac{N^{2}+2}{3} & : x=y \\
\frac{2 \pi^{2}}{N^{2}}(-)^{(x-y) / a} \frac{1}{\sin ^{2}(\pi(x-y) /(N a))} & : x \neq y
\end{array} .\right.
$$

Although it was analysed [54] that this prescription will lead to a non-covariant and non-local continuum limit in lattice QED it can be proven [42, 55] that for scalar theories in one or two dimensions with Yukawa interactions a local renormalisable continuum limit is reached. For that reason the SLAC derivative is used here to regularise the supersymmetric quantum mechanics on the lattice with corresponding action

$$
S=-\sum_{x, y} \frac{1}{2} \hat{\phi}_{x}\left(\hat{\partial}^{\mathrm{SLAC}}\right)_{x y}^{2} \hat{\phi}_{y}+\frac{1}{2} \sum_{x} P\left(\hat{\phi}_{x}\right)^{2}+\sum_{x, y} \bar{\psi}_{x}\left(\hat{\partial}_{x y}^{\mathrm{SLAC}}+P^{\prime}\left(\hat{\phi}_{x}\right) \delta_{x y}\right) \psi_{y}
$$

on lattices with an even number of sites, where field $\hat{\phi}$ and derivative $\hat{\partial}^{\text {SLAC }}$ are dimensionless and arise from a rescaling of the dimensionful quantities with the lattice spacing 9

\footnotetext{
${ }^{7}$ For a phase with unbroken supersymmetry in models with $\Delta=0$ only one specific ground state belongs to the physical spectrum and periodic boundary conditions may be imposed.

${ }^{8}$ In contrast to the operator formalism where the field space is discretised, the lattice path integral is based on a discretisation in the Euclidean time.

${ }^{9}$ The fermionic fields $\psi, \bar{\psi}$ are already dimensionless and do not need to be rescaled.
} 

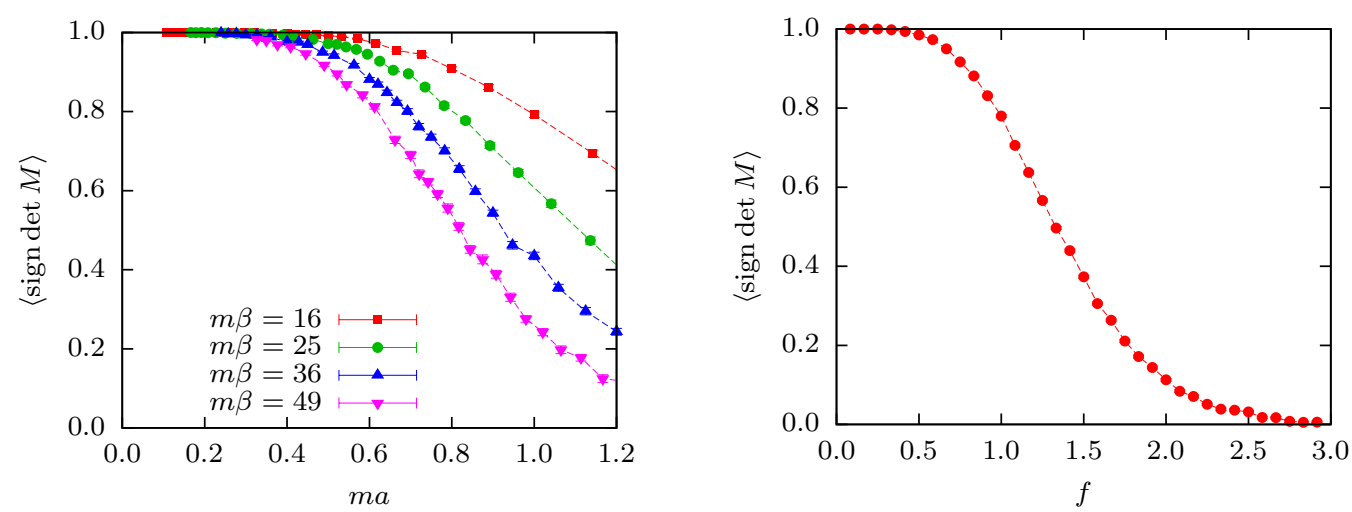

Figure 4: Sign of the fermion determinant measured at fixed $f=1$ (left panel, $10^{6}$ configurations per data point) and at fixed $m \beta=36$ and $N=50$ lattice points (right panel, $5 \cdot 10^{6}$ configurations per data point).

Access to the non-perturbative sector of the lattice model is gained from Monte-Carlo simulations which have become a powerful tool due to increasing computer power and algorithmic improvements that allow for the inclusion of dynamical fermions in simulations. These statistical methods are based on importance sampling and the interpretation of the lattice regularised path integral

$$
\mathcal{Z}=\int \mathrm{D} \hat{\phi} \mathrm{D} \psi \mathrm{D} \bar{\psi} \mathrm{e}^{-S[\hat{\phi}, \psi, \bar{\psi}]}
$$

as probability distribution. To construct the probability density the action is then split into a bosonic and fermionic part according to

$$
S[\hat{\phi}, \psi, \bar{\psi}]=S_{\mathrm{B}}[\hat{\phi}]+\sum_{x, y} \bar{\psi}_{x} M_{x y}[\hat{\phi}] \psi_{y}
$$

Applying the rules of Grassmann integration the fermionic part of the path integral can be integrated out and yields

$$
\mathcal{Z}=\int \mathrm{D} \hat{\phi} \operatorname{det} M[\hat{\phi}] \mathrm{e}^{-S_{\mathrm{B}}[\hat{\phi}]}
$$

In this way (bosonic) expectation values are computed by

$$
\langle\mathcal{O}[\hat{\phi}]\rangle=\mathcal{Z}^{-1} \int \mathrm{D} \hat{\phi} \mathcal{O}[\hat{\phi}] \operatorname{det} M[\hat{\phi}] \mathrm{e}^{-S_{\mathrm{B}}[\hat{\phi}]}
$$

In a Monte-Carlo simulation the lattice regularised fields $\hat{\phi}$ are generated according to the distribution

$$
\rho[\hat{\phi}]=\mathrm{e}^{-S_{\mathrm{B}}[\hat{\phi}]+\ln |\operatorname{det} M[\hat{\phi}]|} .
$$

After a number of $N_{\mathrm{MC}}$ samples one obtains a time series $\hat{\phi}^{(k)}, k=1, \ldots, N_{\mathrm{MC}}$, and expectation values are evaluated using

$$
\langle\mathcal{O}\rangle \stackrel{N_{\mathrm{MC}} \rightarrow \infty}{=} N_{\mathrm{MC}}^{-1} \sum_{k=1}^{N_{\mathrm{MC}}} \mathcal{O}\left[\hat{\phi}^{(k)}\right] .
$$

This expression is only exact iff $\operatorname{det} M \geq 0$. If $\operatorname{det} M$ is negative the sign has to be taken into account by reweighing. However, the emphasis shall lie on the physical questions and for further simulation details the reader is referred to the rich literature on Monte-Carlo methods, e.g. [56-61].

\subsubsection{Sign of the fermion determinant}

For periodic fermionic boundary conditions $\mathcal{Z}_{\mathrm{p}} \propto \Delta$ will vanish in the continuum and a severe sign problem is expected to arise in reweighed expectation values. For thermal boundary conditions it is a priori unknown if there are 

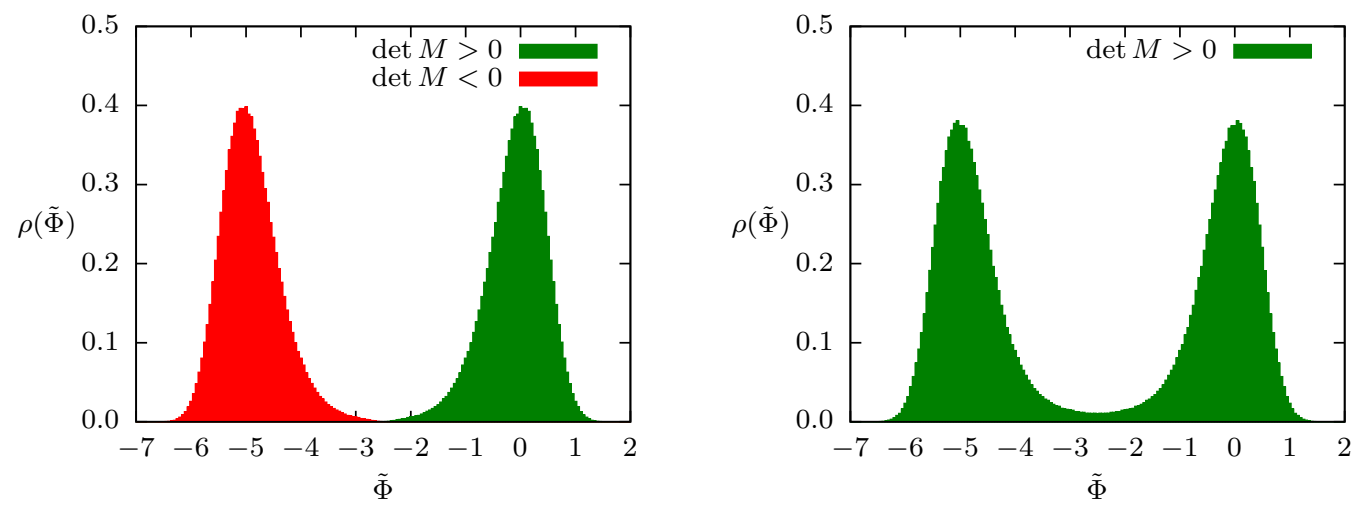

Figure 5: The distribution of the averaged field $\tilde{\Phi}$ for $m \beta=4$ at coupling $f=0.2$ with respect to the sign of the determinant for periodic (left panel, $N=101$ ) and antiperiodic (right panel, $N=100$ ) fermionic boundary conditions obtained from $10^{6}$ configurations.

configurations with $\operatorname{det} M<0$ and if there is any dependence on lattice spacing, temperature, or coupling. For that reason $\langle\operatorname{sign} \operatorname{det} M\rangle$ has been measured in the sign quenched ensemble with the distribution given by Eq. (30) for different parameter sets (see Fig. (4). These results imply a complete absence of the sign problem in the continuum limit for every coupling and temperature. The sign problem only exists for large couplings $f$ at fixed lattice spacing and temperature.

\subsubsection{Ground state structure}

With thermal as well as supersymmetry preserving (periodic) boundary conditions for low temperature $T=0.25$ the ground state structure is analysed 10 Simulations at $f=0.2$ are performed and the distribution of the lattice averaged field $\tilde{\Phi}=N^{-1} \sum_{x} \Phi_{x}$ is analysed with respect to the sign of $\operatorname{det} M$ (see Fig. 5). Configurations with $\tilde{\Phi}>-1 /(2 f)$ are unaffected by a change of boundary conditions whereas the sign of $\operatorname{det} M$ changes for $\tilde{\Phi}<-1 /(2 f)$. This behaviour can be seen explicitly on the level of the discretised action. For the chosen prepotential bosonic and fermionic ground state are related by a $\mathbb{Z}_{2}$ symmetry $\Phi_{x} \rightarrow-\Phi_{x}-1 / f$. $S_{\mathrm{B}}$ is invariant under the symmetry operation whereas the effect on the fermionic contribution depends on the derivative used. The SLAC derivative has an antisymmetric matrix representation, $\partial_{x y}^{\text {SLAC }}=-\partial_{y x}^{\text {SLAC }} \cdot P^{\prime}(\phi)$ enters on the diagonal of the fermion matrix $M$. Applying the $\mathbb{Z}_{2}$ symmetry gives $P^{\prime}(\phi) \rightarrow-P^{\prime}(\phi)$ and changes the sign of the diagonal elements of the fermion matrix. Altogether, the symmetry operation changes $M(\hat{\phi}) \rightarrow-M^{\top}(\hat{\phi})$. For antiperiodic (periodic) fermions the fermion matrix size will be even (odd, respectively) and the determinant will keep the modulus but changes its sign for periodic boundary conditions while for antiperiodic fermions the sign is preserved. Therefore periodic SLAC fermions imply $\mathcal{Z}_{\mathrm{p}}=0$ exactly. The boundary condition dependence of the distribution coincides with introducing $(-1)^{N_{\mathrm{F}}}$ into the path integral for periodic boundary conditions and configurations with $\tilde{\Phi}>-1 /(2 f)$ correspond to the bosonic ground state whereas the other ones correspond to the fermionic ground state, respectively. This is in accordance with results from the operator formalism shown in Fig. 3

\subsubsection{Thermal field distribution}

At finite temperature the single site distribution $\rho_{T}(\Phi)$ of Eq. (14) is computed on a lattice with $N=100$ points at fixed coupling $f=0.2$ in the temperature range $m \beta \in[0.2,4]$ with $10^{6}$ configurations. Even for this coarse lattice the results match almost perfectly with the reference values from the diagonalised Hamiltonian (see Fig. 6 , left panel). At low temperature $m \beta=16$ it is possible to extract the probability distribution in the bosonic ground state by considering only configurations with $\tilde{\Phi}>-1 /(2 f)$. Even at finite (but small) temperature the so-extracted probability distribution follows the exact result at $T=0$, see Fig. 6 (right panel). In consequence, for $T \rightarrow 0$ a thermal mixture of bosonic and fermionic ground state is found whereas at exactly vanishing temperature the system can be triggered to stay in one ground state.

\footnotetext{
${ }^{10}$ Periodic boundary conditions have only been used for this particular analysis of the ground state structure.
} 

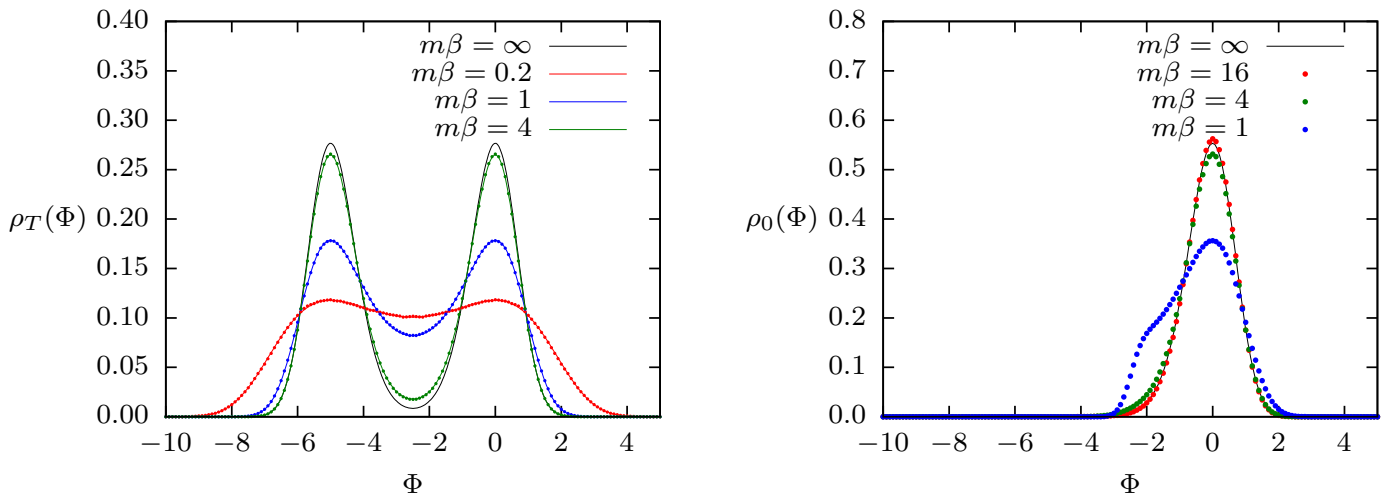

Figure 6: Probability distribution $\rho(\Phi)$ for different temperatures at coupling $f=0.2$ on a $N=100$ lattice. Lines depict the exact results, points arise from the lattice calculations. For comparison the distribution at $T \rightarrow 0$ is also drawn. Left panel: Thermal distribution. Right panel: Exact distribution for the bosonic ground state and distribution measured on configurations with $\tilde{\Phi}>-\frac{1}{2 f}$.

\subsubsection{Effective potential}

The effective potential as introduced in Sec. 2.1.2 is not directly accessible in lattice simulations. A better suited quantity is given by the constraint effective potential [62]

$$
U\left(\tilde{\Phi}_{0}\right)=-\frac{1}{\beta} \ln \left(\int \mathrm{D} \hat{\phi} \operatorname{det} M[\hat{\phi}] \mathrm{e}^{-S_{\mathrm{B}}[\hat{\phi}]} \delta\left(\tilde{\Phi}-\tilde{\Phi}_{0}\right)\right)
$$

which can be straightforwardly computed on the lattice 11 It has been proven for the case of bosonic scalar theories that the constraint effective potential coincides in the limit of infinite spacetime volume (or here equivalently, vanishing temperature) with the effective potential, $\lim _{m \beta \rightarrow \infty} U(\tilde{\Phi})=\lim _{m \beta \rightarrow \infty} V_{\text {eff }}(\tilde{\Phi})$.

We determined the constraint effective potential for various inverse temperatures $m \beta$ at fixed coupling $f=0.2$ for $N=300$ lattice points. The result is depicted in Fig. 7 together with the effective potential for the full theory and for the bosonic/fermionic sector. To avoid the ambiguities of the unknown additive constant, the minimum of each potential is normalised to 012

It is often stated that the conventional effective potential for a quantum mechanical system is strictly convex. This is true for systems with Hilbert space $L^{2}\left(\mathbb{R}^{d}\right)$. For the supersymmetric system the Hilbert space is $L^{2}(\mathbb{R}) \otimes \mathbb{C}^{2}$ and all linear combination of the bosonic and fermionic ground states have the same energy, which implies a flat region in the effective potential as defined in Eq. 13.

To compute the value of the constraint effective potential even around the peak $\tilde{\Phi}=-1 /(2 f)$, reweighting methods [63] similar to the multicanonical ensemble [64] have been applied. Simulations were performed with a modified action $S_{B}^{\prime}=S_{B}-W(\tilde{\Phi})$, with $W(\tilde{\Phi})$ suitably chosen by iterative refinement to approximate $\beta U(\tilde{\Phi})$ between the two minima and $W(\tilde{\Phi})$ constant in the outer regions. Of course, the configurations must be (re-)weighted after simulation with a factor $\exp (-W(\tilde{\Phi})) 13$

It is apparent that the positions of the minima of $U(\tilde{\Phi})$ correspond to the bosonic and fermionic ground state. However, the constraint effective potential shows no tendency to flatten out towards the conventional effective potential. It rather tends towards

$$
\lim _{m \beta \rightarrow \infty} U(\tilde{\Phi})=\lim _{m \beta \rightarrow \infty} \min \left(V_{\text {eff,B }}(\tilde{\Phi}), V_{\text {eff,F }}(\tilde{\Phi})\right),
$$

where $V_{\text {eff,B/F }}$ denotes the effective potential of the bosonic (fermionic, respectively) sector of the Hamiltonian (3).

This result can be explained by the behaviour of fermions on the lattice under a change of boundary conditions. The ratio of the fermion determinant between periodic and antiperiodic boundary conditions can be computed in the

\footnotetext{
${ }^{11}$ Only an additive normalisation constant of the constraint effective potential is left undetermined.

${ }^{12}$ This amounts to an offset for the effective potential of the full theory that is given by the ground state energy, $\Delta V_{\text {eff }}=E_{0}=3.68 \cdot 10^{-5} m$.

${ }^{13}$ In a conventional Monte-Carlo simulation without reweighting the configurations around the peak will be suppressed at $m \beta=49$ by more than $\mathrm{e}^{-20}$.
} 


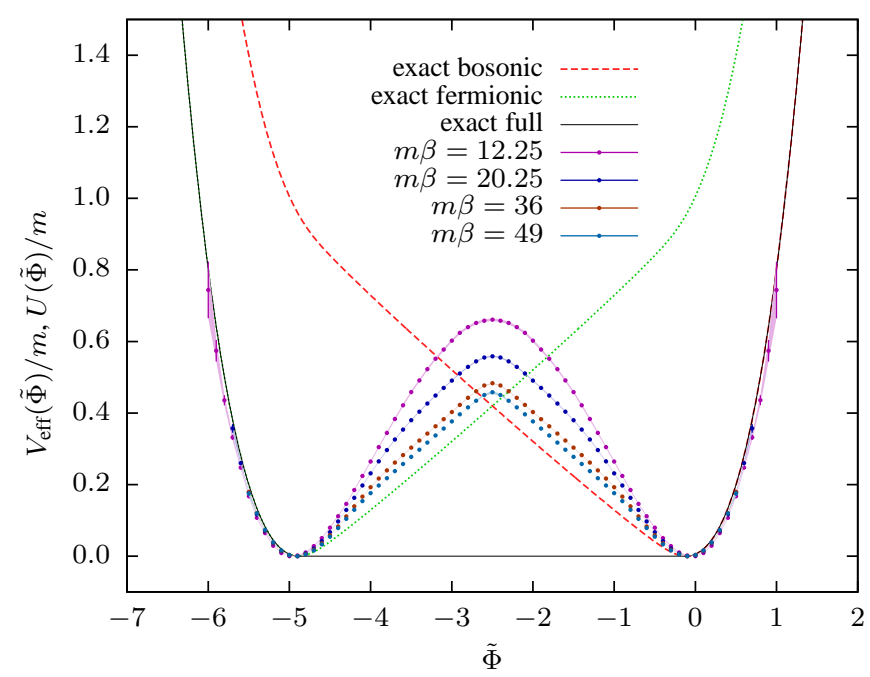

Figure 7: Effective potential for the full model ("exact full"), bosonic sector ("exact bosonic"), and fermionic sector ("exact fermionic") of the Hamiltonian at $T=0$ and constraint effective potential from lattice simulations of the full model at various $m \beta$ for coupling $f=0.2$ and $N=300$ lattice points, measured with up to $10^{7}$ configurations.

continuum limit [65], and by using zeta function regularisation [66] one gets rid of the free determinant as normalisation constant. The continuum result

$$
R_{\beta}[\phi] \equiv \frac{\operatorname{det} M_{\mathrm{p}}[\phi]}{\operatorname{det} M_{\mathrm{ap}}[\phi]}=\frac{\sinh \left[\frac{1}{2} \int_{0}^{\beta} P^{\prime}(\phi(\tau)) \mathrm{d} \tau\right]}{\cosh \left[\frac{1}{2} \int_{0}^{\beta} P^{\prime}(\phi(\tau)) \mathrm{d} \tau\right]}
$$

will be either +1 or -1 in the infinite volume limit, depending on the value of $\tilde{\Phi}$,

$$
\lim _{\beta \rightarrow \infty} R_{\beta}(\phi)=\left\{\begin{array}{ll}
+1 & : \tilde{\Phi}>-\frac{1}{2} f^{-1} \\
-1 & : \tilde{\Phi}<-\frac{1}{2} f^{-1}
\end{array},\right.
$$

which is equivalent to $(-1)^{N_{\mathrm{F}}}$. I.e by means of the lattice path integral with fixed (thermal) boundary conditions it is only possible to assess either the zero fermion sector or the one fermion sector. The interpolating states between both sectors that are responsible for the flattening of the effective potential are thus not accessible by the (lattice) path integral at any finite temperature.

\subsubsection{Two-point functions and spectrum}

Lattice based path integral methods provide a non-perturbative way to gain information about the spectrum of the theory. Via the long distance behaviour of correlators $C(t) \stackrel{t \rightarrow \infty}{\longrightarrow} \exp \left(-m_{\text {phys }} t\right)$ it is possible to extract the physical 'pole mass' $m_{\text {phys }}$ which is given by the imaginary part of the pole of the propagator $G(p)=(\mathcal{F} C)(p)$, the Fourier transform of the correlator, and describes the energy difference between the ground state and first excited state of the theory. For that reason connected correlation functions in the thermal ensemble have been computed for bosonic and fermionic fields (see Fig. 8) with

$$
C_{\mathrm{B}}(t)=\langle\Phi(t) \Phi(0)\rangle-\langle\Phi\rangle^{2} \quad \text { and } \quad C_{\mathrm{F}}(t)=\langle\psi(t) \bar{\psi}(0)\rangle .
$$

The correlators take non-vanishing constant values for large distances in a region where the exponential falloff drops below the visibility scale 14 Correlators computed from the lattice regularised theory fit nicely to the ones computed

\footnotetext{
${ }^{14}$ This is not directly related to any unconnected part. Here only connected correlators are considered.
} 

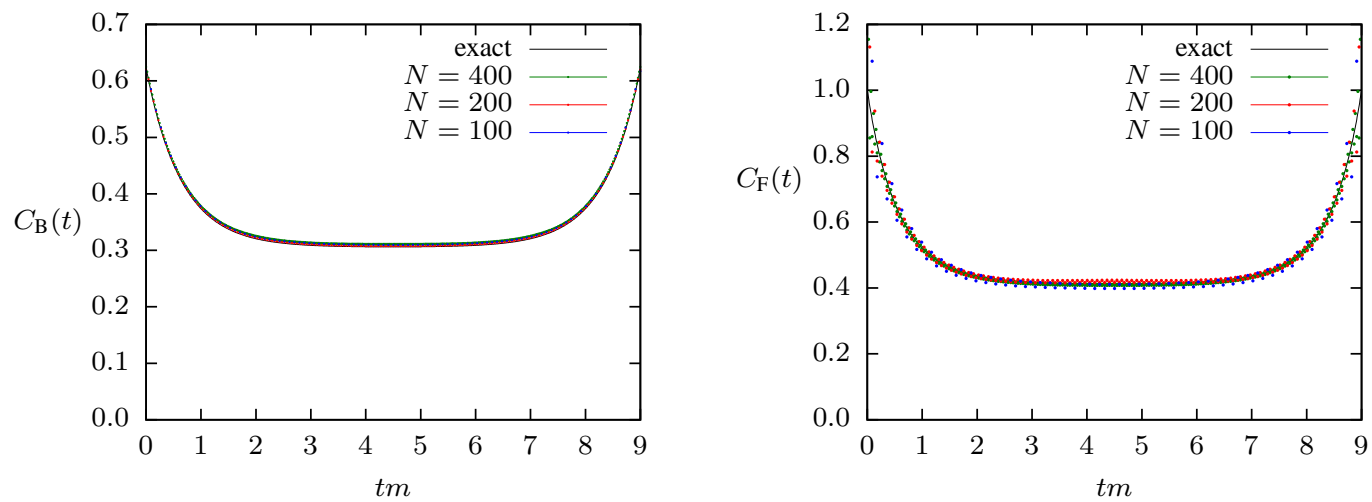

Figure 8: The two-point function (left panel: bosonic, right panel: fermionic) for the thermal ensemble given by $m \beta=9$ and coupling $f=1$ computed by the diagonalised Hamiltonian and on a lattices with $N \in\{100,200,400\}$ points. For the bosonic case the data points coincide with the reference values. A statistics of up to $10^{7}$ configurations has been used.
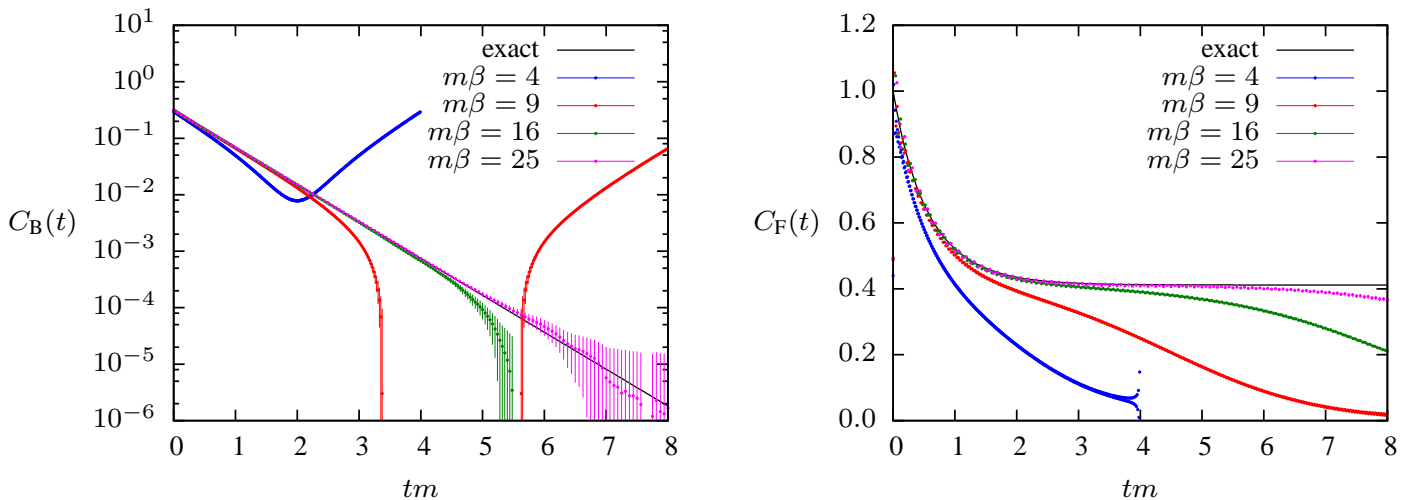

Figure 9: Bosonic (left panel) and fermionic (right panel) correlator in the ensemble projected to one ground state as obtained from the diagonalised Hamiltonian and from lattice simulations with $N=400$ sites at coupling $f=1$ and statistics of up to $3 \cdot 10^{7}$ configurations.

by the diagonalised Hamiltonian. Fluctuation are still visible around the continuum values with the size of fluctuations vanishing for smaller lattice spacings. Further it is possible from the fermionic correlator at small (but non-vanishing) temperature to compute the overlap of bosonic and fermionic ground state $\left|\left\langle 0_{\mathrm{F}}\left|\Psi^{\dagger}\right| 0_{\mathrm{B}}\right\rangle\right|^{2}=0.41174$ by the approximation $C_{\mathrm{F}}(m \beta / 2)=0.419(7)$ for $m \beta=16$ and $N=400$.

With nearly vanishing temperature the system will mainly reside in the ground states, and with the results of Sec. 2.2.2 it is possible to compute the correlation function at $T=0$ by projecting to one of the ground states (see Fig. 9). In that case the bosonic correlator shows no constant part and the exponential behaviour completely coincides with the one resulting from the first excited (bosonic) state. From the lattice values at $N=400$ a mass of $m_{\text {phys }}=1.5047(15) m$ is extracted through an exponential fit in the range $t m \in[1,3]$ from a simulation at $m \beta=25$. This is very close to the exact value $E_{1}-E_{0}=1.5046 \mathrm{~m}$. Of course this method is only applicable for $t<\beta / 2$ and there will be large deviations for $t$ close to $\beta / 2$.

All these results clearly demonstrate that it is possible to extract correlators at finite and zero temperature from a lattice discretisation in complete agreement with exact results in the continuum limit. The degenerate ground states are visible as a constant part in the fermionic correlator and a projection to one ground state allows to extract the energy difference between the ground state and first excited state (see Fig.10). 


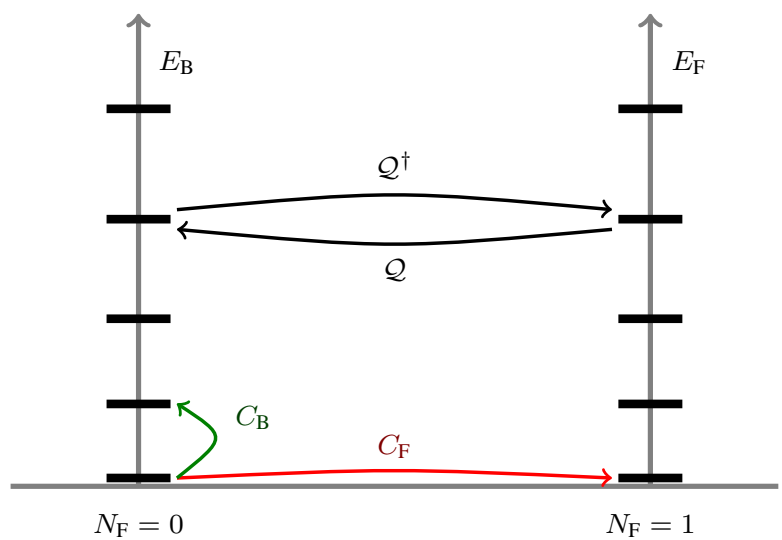

Figure 10: Excitations visible as $t \rightarrow \infty$ behaviour of the correlators evaluated on configurations projected to the bosonic ground state.

\begin{tabular}{lcc}
\hline$T^{-1}$ & exact & extrapolated \\
\hline 1.00 & 0.4392039 & $0.43902(18)$ \\
1.44 & 0.4027741 & $0.40298(20)$ \\
4.00 & 0.3730929 & $0.37327(18)$ \\
\hline
\end{tabular}

Table 1: Linearly extrapolated lattice values of the Ward identity $\langle P\rangle / \sqrt{m}$ in comparison to the exactly calculable ones from the operator formalism for three different temperatures $T$ at coupling $f=1$.

\subsubsection{Ward identity}

For field transformations $\Phi^{\prime}=\Phi+\delta \Phi$ that do not change the path integral measure $15\left(\mathcal{D} \Phi=\mathcal{D} \Phi^{\prime}\right)$ Ward identities naturally arise on the level of observables as

$$
\langle\mathcal{O}\rangle=\mathcal{Z}^{-1} \int \mathcal{D} \Phi^{\prime} \mathcal{O}\left[\Phi^{\prime}\right] \mathrm{e}^{-S\left[\Phi^{\prime}\right]}=\mathcal{Z}^{-1} \int \mathcal{D} \Phi(\mathcal{O}[\Phi]+\delta \mathcal{O}[\Phi])(1-\delta S[\Phi]) \mathrm{e}^{-S[\Phi]}=\langle\mathcal{O}+\delta \mathcal{O}-\mathcal{O} \delta S\rangle
$$

implying $\langle\delta \mathcal{O}\rangle=\langle\mathcal{O} \delta S\rangle$. If further the action and ground state are invariant under the transformation given by $\delta$ then $\langle\delta \mathcal{O}\rangle$ vanishes for every observable $\mathcal{O}$.

For unbroken supersymmetric theories Ward identities are used to test the supersymmetry restoration in the continuum limit by analysing the continuum limit of $\langle\delta \mathcal{O}\rangle$ for a given observables $\mathcal{O}$. If supersymmetry is broken then Ward identities will not be fulfilled in the continuum limit, $\langle\delta \mathcal{O}\rangle \neq 0$. On the lattice supersymmetry will be further broken explicitly by a finite lattice spacing and by finite temperature.

A simple Ward identity is provided by

$$
\int \mathrm{d} t\left\langle\delta^{(1)} \bar{\psi}\right\rangle=-\bar{\varepsilon} \int \mathrm{d} t\langle\dot{\phi}+P(\phi)\rangle=-\bar{\varepsilon}\left\langle\int \mathrm{d} t P(\phi)\right\rangle=-\beta \bar{\varepsilon}\langle P(\phi)\rangle,
$$

and a dimensionless identity is given by $\langle P\rangle / \sqrt{m}=0$ iff the ground state is invariant under the supersymmetry. The impact of the explicit supersymmetry breaking on the continuum result at vanishing temperature is depicted in Fig. 11 for coupling $f=1$. The finite $a$ effects are small for the considered lattice spacings and a linear extrapolation to $a=0$ provides results in full agreement with the continuum results (see Tab. 1). Finite temperature effects are negligible for $T<0.2$. The numerically exact value from the operator formalism is $\langle P\rangle / \sqrt{m}=0.37251$ and corresponds to the non-supersymmetric ground states. This quantity is invariant under the $\mathbb{Z}_{2}$ symmetry $\Phi \rightarrow \Phi-1 / f$ and is not sensitive to the specific ground state chosen at $T=0$.

\footnotetext{
${ }^{15}$ Here only the anomaly free case is considered. If the path integral measure is changed under the transformation additional contributions must be taken into account.
} 

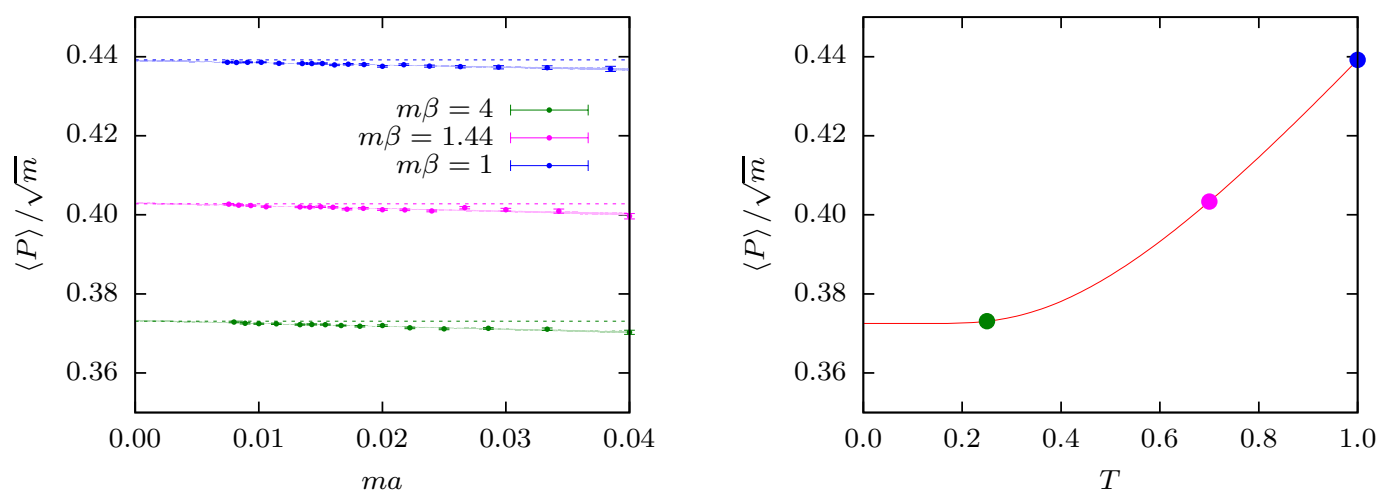

Figure 11: Prepotential for coupling $f=1$. Left panel: Continuum limit of the prepotential $\langle P\rangle / \sqrt{m}$ which serves as simplest Ward identity for the supersymmetric quantum mechanics. (The dashed lines denote the continuum values while the shaded area gives the error bound of the linear extrapolation to $a=0$.) Right panel: Temperature dependence of of $\langle P\rangle / \sqrt{m}$ computed from the diagonalised Hamiltonian (data from the left panel is marked by points).

\section{3. $\mathcal{N}=1$ Wess-Zumino model}

Supersymmetry as incorporated in the minimal supersymmetric standard model [12] or extensions thereof [67] can only be a fundamental symmetry of nature if it is spontaneously broken on experimentally accessible energy scales with a phase transition at a much higher energy. Guided by this observation there is a need to study supersymmetry breaking phase transitions with non-perturbative tools. To explore the possibilities provided by lattice regularisations a minimal model is chosen, namely the $\mathcal{N}=(1,1)$ Wess-Zumino model in two dimensions [68]. It is obtained from the $\mathcal{N}=(2,2)$ Wess-Zumino model, which provides a dimensionally reduced version of the matter sector of the minimal supersymmetric standard model, by constraining the fields of the $\mathcal{N}=(2,2)$ model to be real [42, 46, 60, 69-71]. This amounts to turning complex scalars into real ones and replacing Dirac fermions with Majorana fermions, leading to a minimal field content with only one bosonic and fermionic degree of freedom 16 For models with short range interactions at least two dimensions are necessary to observe a phase transition. In particular supersymmetric quantum mechanics is not sufficient to model a phase transition since the systems reside in one specific phase depending on the highest power of the superpotential (cf. Sec. 2.1). Since the seminal work by Witten [15] it is known that the index $\operatorname{Tr}(-1)^{N_{\mathrm{F}}}$ can vanish for specific choices of the prepotential and supersymmetry may be broken spontaneously depending on the couplings of the prepotential for the $\mathcal{N}=1$ Wess-Zumino model.

The off-shell continuum formulation is given by the action

$$
S=\int \mathrm{d}^{2} x \frac{1}{2}\left(\left(\partial_{\mu} \phi\right)^{2}+\bar{\psi}\left(\not \partial+P^{\prime}(\phi)\right) \psi+2 F P(\phi)-F^{2}\right),
$$

where $F$ and $\phi$ denote real scalar fields and $\psi$ is a (real) Majorana spinor with two spinorial components. This formulation is invariant under the supersymmetry transformations

$$
\delta \phi=\bar{\epsilon} \psi, \quad \delta \psi=(\not \phi \phi-F) \epsilon, \quad \delta F=-\bar{\epsilon} \not \partial \psi .
$$

By eliminating the auxiliary field $F=P(\phi)$ one arrives at the on-shell action

$$
S=\int \mathrm{d}^{2} x \frac{1}{2}\left(\left(\partial_{\mu} \phi\right)^{2}+\bar{\psi}\left(\not \partial+P^{\prime}(\phi)\right) \psi+P(\phi)^{2}\right) .
$$

Here, the prepotential $P$ is chosen to be

$$
P(\phi)=\frac{\mu_{0}^{2}}{\sqrt{2 \lambda}}+\sqrt{\frac{\lambda}{2}} \phi^{2},
$$

\footnotetext{
${ }^{16}$ From a practical point of view the absence of gauge fields has the advantage that derivatives can be applied in momentum space to speed up Monte-Carlo simulations considerably.
} 


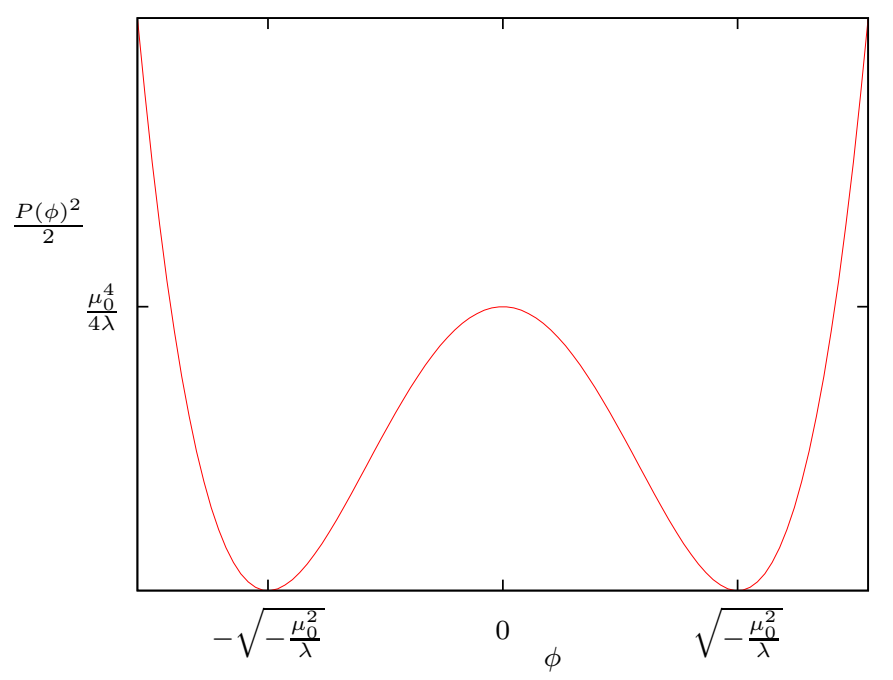

Figure 12: The classical bosonic potential of the Wess-Zumino model given in Eq. 43.

so that the classical potential (Fig. 12) for the scalar part corresponds to a $\phi^{4}$ theory with $\mathbb{Z}_{2}$ symmetry $(\phi \rightarrow-\phi)$,

$$
\frac{P(\phi)^{2}}{2}=\frac{\mu_{0}^{2}}{2} \phi^{2}+\frac{\lambda}{4} \phi^{4}+\text { const. }
$$

while the fermions acquire a Yukawa interaction. An analysis of the Witten index [15] reveals one bosonic and one fermionic ground state that imply $\operatorname{Tr}(-1)^{N_{\mathrm{F}}}=0$. This ground state structure then allows for a spontaneous supersymmetry breaking. It is expected that for fixed $\lambda>0$ and $\mu_{0}^{2} \ll 0$ the system cannot tunnel between the two ground states so that supersymmetry is unbroken. On the other hand, for $\mu_{0}^{2}>0$ both ground state energies are lifted above zero and supersymmetry is broken. In consequence there will be a supersymmetry breaking phase transition for some $\mu_{0}^{2}<0$ and this section is devoted to the study of this transition.

There have already been several studies aiming at analysing and understanding supersymmetry breaking in this model. By a strong coupling expansion [72] the occurrence of a supersymmetry breaking phase transition was predicted. Calculations of the ground state energy with Monte-Carlo methods [73] confirm this expectation. Afterwards lower bounds on the ground state energy have been analysed [74, 75] to obtain a phase diagram of supersymmetry breaking by working in the Hamiltonian formalism and making a numerical analysis with Green's function MonteCarlo methods. However, the obtained critical lattice couplings so far are only unrenormalised couplings corresponding to one specific lattice spacing. Recently an analysis based on exact renormalisation group methods [76] has been performed [77] where no supersymmetry breaking is introduced during the renormalisation group flow. Again, the phase diagram has been obtained and supersymmetry breaking is found to coincide with a restoration of the $\mathbb{Z}_{2}$ symmetry in a second order phase transition. A supersymmetric (massive) phase is found for weakly coupled systems as well as a phase of broken supersymmetry with goldstinos, the massless Goldstone fermions [78] of the broken supersymmetry 17 , and bosons whose mass vanishes with growing renormalisation group scale 18 But still the critical coupling depends on the chosen regulator which prevents a direct comparison of numerical values.

In general a naive discretisation of a lattice action has to face the problem of broken supersymmetry for finite lattice spacing with the need to fine-tune lattice couplings to reach a supersymmetric continuum limit. However, this model has the advantage that the necessary counterterms have been analysed in lattice perturbation theory [37] and a lattice prescription is given that ensures the supersymmetric continuum limit (perturbatively). Simulations of the given discretisation (based on the Wilson derivative) have already been performed [80] and a tunnelling between the possible ground states is found to coincide with the onset of supersymmetry breaking and the appearance of a

\footnotetext{
${ }^{17}$ This breaking is not forbidden by the Mermin-Wagner theorem [79] that only applies to bosonic symmetry generators.

${ }^{18}$ Also the corresponding critical exponents have been determined in [77].
} 


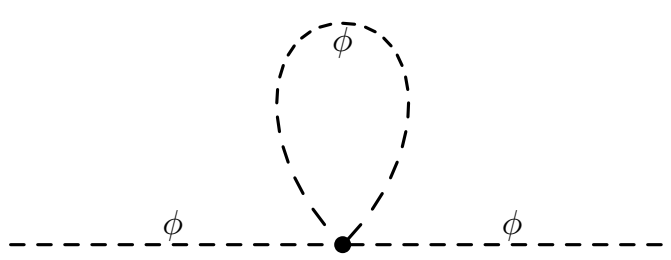

Figure 13: The only divergent Feynman diagram for the bosonic $\phi^{4}$ model in the $\mathbb{Z}_{2}$ symmetric phase.

goldstino. Despite all these successes several open issues remain: The breaking of a $\mathbb{Z}_{2}$ symmetry (which is correlated with the restoration of supersymmetry) has so far only been analysed with a $\mathbb{Z}_{2}$ breaking action 19 Further the given critical coupling is still regulator dependent and not directly comparable to other methods. For that reason the aim of this section is the non-perturbative determination from first principles of a renormalised critical coupling in the continuum limit.

\subsection{Quenched model}

Although our primary focus lies on the supersymmetric model it is useful to exemplify the definition of a renormalised critical coupling in the setting of the quenched model where fermionic contributions are neglected. This becomes especially important because a non-standard discretisation based on the SLAC derivative is used.

With the prepotential of Eq. (42) the quenched model is nothing but the two dimensional $\phi^{4}$ model with action

$$
S_{\mathrm{B}}=\int \mathrm{d}^{2} x \frac{1}{2}\left(\left(\partial_{\mu} \phi\right)^{2}+\mu_{0}^{2} \phi^{2}+\frac{\lambda}{2} \phi^{4}\right) .
$$

This model is (classically) invariant under a discrete $\mathbb{Z}_{2}$ symmetry $(\phi \rightarrow-\phi)$ which can be broken dynamically in the full quantum theory [81]. The symmetric phase is characterised by $\langle\phi\rangle=0$, whereas in the broken phase (in the thermodynamic limit) $\langle\phi\rangle \neq 0$.

In contrast to the full $\mathcal{N}=2$ Wess-Zumino model the $\phi^{4}$ model (as well as the full $\mathcal{N}=1$ Wess-Zumino model) is not finite and there is need for a renormalisation of couplings. In the $\mathbb{Z}_{2}$ symmetric phase the only divergence arises from the 'leaf' diagram (see Fig. 13) and the model can be made finite with a mass renormalisation,

$$
S_{\mathrm{B}}=\int \mathrm{d}^{2} x \frac{1}{2}\left(\left(\partial_{\mu} \phi\right)^{2}+\mu^{2} \phi^{2}+\frac{\lambda}{2} \phi^{4}-\delta \mu^{2} \phi^{2}\right) .
$$

The (one-loop) relation between the inverse propagators is given in terms of renormalised couplings by

$$
G^{-1}(p)=p^{2}+\mu^{2}+\Sigma\left(p^{2}\right), \quad \Sigma\left(p^{2}\right)=3 \lambda A_{\mu^{2}}-\delta \mu^{2}, \quad A_{\mu^{2}}=\int \frac{\mathrm{d}^{2} p}{(2 \pi)^{2}} \frac{1}{p^{2}+\mu^{2}}
$$

and the only (logarithmically) divergent contribution is $A_{\mu^{2}}$. Hence all ultraviolet divergences can be removed by a renormalisation of the mass term 20

$$
\delta \mu^{2}=3 \lambda A_{\mu^{2}} \quad \Leftrightarrow \quad \mu_{0}^{2}=\mu^{2}-3 \lambda A_{\mu^{2}} .
$$

This renormalisation prescription will also be sufficient in the $\mathbb{Z}_{2}$ broken phase [81]. We follow [82] and use the dimensionless renormalised coupling $f=\lambda / \mu^{2}$ that distinguishes between the symmetric and broken phase. By contrast, (naively) possible definitions of a renormalised coupling that are based on the pole mass or the propagator at vanishing momentum are not sufficient to distinguish between both phases. Note that this renormalisation prescription can only be applied in a given scheme and continuum results will then follow by removing the ultraviolet regulator 21

\footnotetext{
${ }^{19}$ The Wilson term for the fermionic part of the action will break the $\mathbb{Z}_{2}$ symmetry as analysed in [60].

${ }^{20}$ Equivalently this renormalisation can be obtained by a normal ordering of the interaction part in the symmetric phase,$$
S_{\mathrm{B}}=\int \mathrm{d}^{2} x \frac{1}{2}\left(\left(\partial_{\mu} \phi\right)^{2}+\mu^{2} \phi^{2}+\frac{\lambda}{2}: \phi^{4}: \mu\right),
$$

where : . : $\mu$ denotes normal ordering with respect to the mass $\mu$.

${ }^{21}$ There is no need to introduce an infrared regulator.
} 


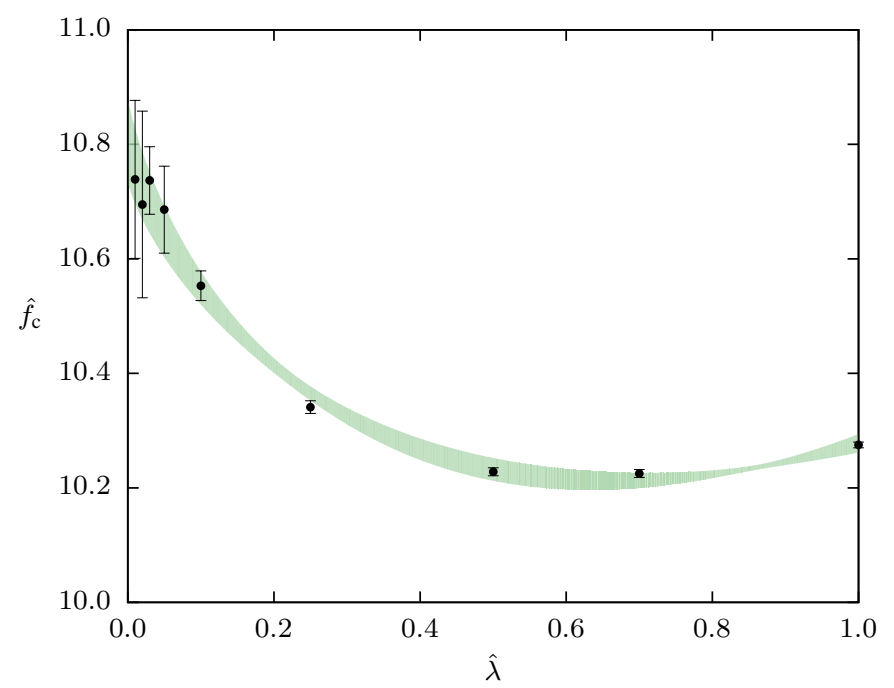

Figure 14: Extrapolation of the critical couplings of the bosonic $\phi^{4}$ model to the continuum limit. The shaded region indicates error bands for the extrapolation using the functional form [52]. Data points are taken from [83] with the canonical discretisation while confidence bands are computed here.

\subsubsection{The $\mathbb{Z}_{2}$ phase transition on the lattice}

The computation of the critical coupling in the bosonic $\phi^{4}$ model has a long history where several methods and approximations (e.g. based on the Gaussian effective potential or light-cone quantisation) have contributed. For a recent overview containing the most precise Monte-Carlo results see [83]. Since these accurate results serve as reference values in the present work we briefly recall the most important points. The lattice regularisation in [82, 83] is based on the "canonical" discretisation utilising the forward derivative and a renormalised critical coupling is computed with high precision. The canonical model is given by

$$
S_{\mathrm{B}}=\sum_{x} \frac{1}{2}\left(\sum_{\nu}\left(\phi_{x+\hat{\nu}}-\phi_{x}\right)^{2}+\hat{\mu}_{0}^{2} \phi_{x}^{2}+\frac{\hat{\lambda}}{2} \phi_{x}^{4}\right)
$$

with dimensionless lattice parameters $\hat{\lambda}=\lambda a^{2}$ and $\hat{\mu}_{0}^{2}=\mu_{0}^{2} a^{2}$. Since $\lambda$ acquires no renormalisation it is used to set the scale. Equivalently $\hat{\lambda}$ determines the lattice spacing with $\hat{\lambda} \rightarrow 0$ in the continuum limit. The (dimensionless) renormalised coupling is again given by $\hat{f}=\hat{\lambda} / \hat{\mu}^{2}$ and the corresponding $\hat{\mu}_{0}^{2}$ can be computed via $\hat{\mu}_{0}^{2}=\hat{\mu}^{2}-3 \hat{\lambda} A_{\hat{\mu}^{2}}$, where $A_{\hat{\mu}^{2}}$ that enters the normal ordering is given in the infinite volume limit by the lattice propagator for the forward derivative,

$$
A_{\hat{\mu}^{2}}=\lim _{n \rightarrow \infty} n^{-2} \sum_{k_{1}=1}^{n} \sum_{k_{1}=1}^{n} \frac{1}{\hat{\mu}^{2}+4 \sin ^{2}\left(\pi k_{1} / n\right)+4 \sin ^{2}\left(\pi k_{2} / n\right)} .
$$

Because $A_{\hat{\mu}^{2}}$ diverges only logarithmically for $a \rightarrow 0$ it follows that $\hat{\mu}^{2}, \hat{\mu}_{0}^{2} \rightarrow 0$ in the continuum limit at fixed $\hat{f}$. One can show that at every fixed $\hat{\lambda}$ a second order phase transition appears. However, the continuum physics at these phase transitions corresponds to an infinite continuum coupling $\lambda$ and is therefore only indirectly relevant for the continuum $\phi^{4}$ model under consideration.

At finite lattice spacing (given by fixed $\hat{\lambda}$ ) there will be a $\mathbb{Z}_{2}$ breaking phase transition and a critical $\hat{\mu}_{\mathrm{c}}^{2}$ can be extracted. The renormalised critical coupling in the continuum limit is then determined via

$$
f_{\mathrm{c}}=\left[\frac{\lambda}{\mu^{2}}\right]_{\mathrm{crit}}=\lim _{\hat{\lambda} \rightarrow 0} \hat{f}_{\mathrm{c}} \quad \text { with } \quad \hat{f}_{\mathrm{c}}=\frac{\hat{\lambda}}{\hat{\mu}_{\mathrm{c}}^{2}} .
$$



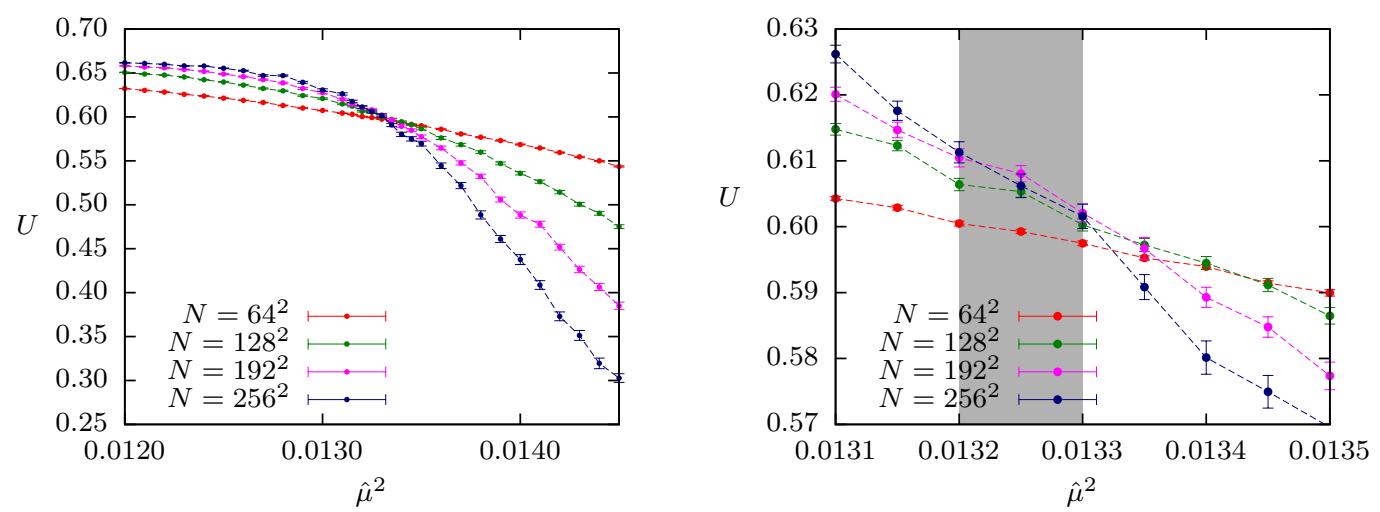

Figure 15: Binder cumulants at $\hat{\lambda}=0.15$ for different lattice sizes. The coarse view is given in the left panel while a close-up view of the critical region is shown in the right panel. From the intersection point for the largest lattices a critical coupling $\hat{f}_{\mathrm{c}}=\frac{0.15}{0.01325(5)}=11.321(43)$ is extracted. The shaded region gives the error band for the infinite volume extrapolation.

The phase transition itself for finite $\hat{\lambda}$ can be determined from the Binder cumulant

$$
U=1-\frac{\left\langle\tilde{\phi}^{4}\right\rangle}{3\left\langle\tilde{\phi}^{2}\right\rangle^{2}} \quad \text { with } \quad \tilde{\phi}=N^{-1} \sum_{x} \phi_{x}
$$

which becomes independent of the lattice volume at the second order phase transition point [84] 22 The critical coupling has been determined from lattices up to a size of $1200^{2}$ and was found to be affected by linear and logarithmic corrections in the lattice spacing. An extrapolation based on the published values in [83] for $\hat{\lambda} \in[0.01,1]$ to the continuum using a functional form

$$
\hat{f}_{\mathrm{c}}(\hat{\lambda}) \approx f_{\mathrm{c}}+a \hat{\lambda}+b \hat{\lambda} \ln \hat{\lambda}
$$

reveals a renormalised critical coupling in the continuum of $f_{\mathrm{c}}=10.81(7)$ (see Fig. 14).

\subsubsection{Regulator independence of the renormalised critical coupling}

The results of Sec. 2 and results in [40, 42, 46, 60, 71] imply that a discretisation based on the SLAC derivative gives results close to the continuum limit and will not break the $\mathbb{Z}_{2}$ symmetry of the full supersymmetric model. For that reason the SLAC derivative will also be used to simulate the $\mathcal{N}=1$ Wess-Zumino model. In contrast to other low dimensional scalar supersymmetric models it is now necessary to cope with a logarithmic mass renormalisation and the renormalised lattice coupling depends on the chosen renormalisation procedure.

To justify the applicability of the SLAC derivative also for the present case simulations based on the lattice action

$$
S=\sum_{x} \frac{1}{2}\left(\sum_{\nu}\left(\partial_{\nu}^{\mathrm{SLAC}} \phi\right)_{x}^{2}+\hat{\mu}_{0}^{2} \phi_{x}^{2}+\frac{\hat{\lambda}}{2} \phi_{x}^{4}\right)
$$

have been performed where the mass parameter is still given by $\hat{\mu}_{0}^{2}=\hat{\mu}^{2}-3 \hat{\lambda} A_{\hat{\mu}^{2}}$. Only now, $A_{\hat{\mu}^{2}}$ is determined from the propagator based on the SLAC derivative,

$$
A_{\hat{\mu}^{2}}=\lim _{n \rightarrow \infty}(2 n)^{-2} \sum_{k_{1}=-n+1}^{n} \sum_{k_{2}=-n+1}^{n} \frac{1}{\hat{\mu}^{2}+\left(\pi k_{1} / n\right)^{2}+\left(\pi k_{2} / n\right)^{2}} .
$$

Similar to the case of the naive discretisation the crossing of the Binder cumulant $U$ for different lattice volumes at fixed $\hat{\lambda}$ determines the critical $\hat{\mu}^{2}$ and therefore the critical coupling $\hat{f}_{\mathrm{c}}$. This procedure is exemplified for $\hat{\lambda}=0.15$

\footnotetext{
${ }^{22}$ Strictly speaking there is still a slight volume dependence such that the large volume extrapolation of the intersection points for different lattice volumes corresponds to the phase transition.
} 


\begin{tabular}{cc}
\hline$\hat{\lambda}$ & $\hat{f}_{\mathrm{c}}$ \\
\hline 0.02 & $11.035(76)$ \\
0.05 & $11.112(74)$ \\
0.10 & $11.268(57)$ \\
0.15 & $11.321(43)$ \\
0.20 & $11.386(42)$ \\
0.25 & $11.429(29)$ \\
\hline
\end{tabular}

Table 2: Renormalised critical couplings for the $\phi^{4}$ model as determined from lattice sizes up to $256^{2}$ with the SLAC derivative.

in Fig. 15 where lattice sizes up to $256^{2}$ were used. As an outcome of these calculations critical couplings have been determined for six different $\hat{\lambda}$ (see Tab.2). Again, an extrapolation to the continuum limit $\hat{\lambda} \rightarrow 0$ has been performed according to Eq. (52) (see Fig. 16) and gives the renormalised critical coupling in the continuum of $f_{\mathrm{c}}=10.92(13)$ which is in complete agreement with the reference value $f_{\mathrm{c}}=10.81(7)$ of [83]. This proves that the lattice $\phi^{4}$ model with non-local SLAC derivative possesses the correct continuum limit, as expected from the analytic studies in [42, 55]. The definition of a renormalised critical continuum coupling is independent of the chosen lattice regulator.

\subsection{Full dynamical model}

By inclusion of dynamical fermions the model is now given in the continuum by the action (41). Irrespective of the chosen prepotential $P(\phi)$ the action is invariant under one supersymmetry. The dynamical breaking of this supersymmetry for the prepotential defined in Eq. (42) will be analysed in the following.

\subsubsection{Renormalised lattice parameters}

Using a discretisation based on the SLAC derivative the lattice action is given by a direct discretisation of the corresponding continuum action,

$$
S=\sum_{x} \frac{1}{2}(\sum_{\nu}\left(\partial_{\nu}^{\mathrm{SLAC}} \phi\right)_{x}^{2}+\hat{\mu}_{0}^{2} \phi_{x}^{2}+\frac{\hat{\lambda}}{2} \phi_{x}^{4}+\sum_{y} \psi_{x}^{\top} \underbrace{C\left(\partial_{x y}^{\mathrm{SLAC}}+\sqrt{2 \hat{\lambda}} \phi_{x} \delta_{x y}\right)}_{=M[\phi]} \psi_{y}) .
$$

In a Majorana representation with $\gamma$ matrices and charge conjugation matrix

$$
\gamma_{0}=\left(\begin{array}{cc}
1 & 0 \\
0 & -1
\end{array}\right), \quad \gamma_{1}=\left(\begin{array}{cc}
0 & -1 \\
-1 & 0
\end{array}\right), \quad C=\left(\begin{array}{cc}
0 & -1 \\
1 & 0
\end{array}\right)
$$

is the fermion matrix $M$ real and antisymmetric.

In [37] a lattice model of the $\mathcal{N}=1$ Wess-Zumino model built upon the symmetric derivative has been analysed. To avoid the species doubling problem a Wilson mass term has been added to the prepotential. Similar discretisations were used for the $\mathcal{N}=2$ Wess-Zumino model in [42, 46, 60, 70, 71]. With properly renormalised coupling parameter (as described below) is was shown that the supersymmetric continuum limit is reached. Although this restoration was only analysed for a discretisation based on Wilson fermions the whole line of argument is directly applicable for the SLAC derivative. This follows from the fact that the SLAC derivative for two dimensional models with Yukawa interaction needs no non-local or non-covariant counterterms to construct a local continuum limit and the lattice degree of divergence of Feynman diagrams coincides with the divergence of the corresponding continuum diagrams [40, 42, 85].

The analysis of divergent diagrams in [37] starts from the $\mathbb{Z}_{2}$ broken phase and shows that a logarithmic renormalisation of the bare mass parameter is necessary to cancel divergent contributions,

$$
\hat{\mu}_{0}^{2}=\hat{\mu}^{2}+\frac{\hat{\lambda}}{4 \pi}\left(\ln \hat{\mu}^{2}+c\right)
$$

where $c$ may be any constant to fix the renormalisation scale. In analogy to the quenched model this constant is now fixed to obtain

$$
\hat{\mu}_{0}^{2}=\hat{\mu}^{2}-1 \hat{\lambda} A_{\hat{\mu}^{2}}
$$




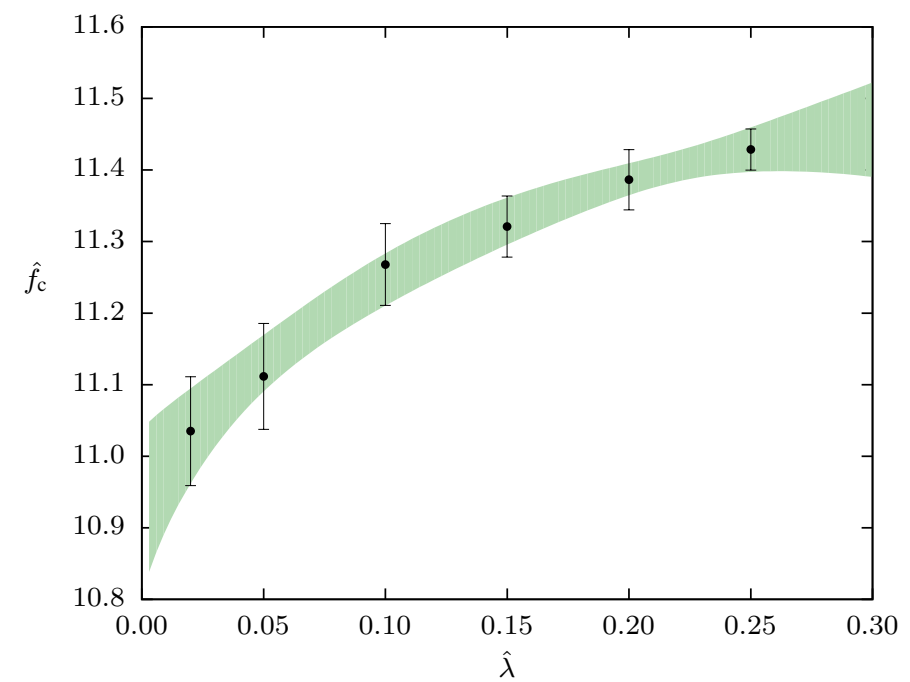

Figure 16: Extrapolation of the critical couplings of the bosonic $\phi^{4}$ model based on the SLAC derivative to the continuum limit according to the functional form 52 .

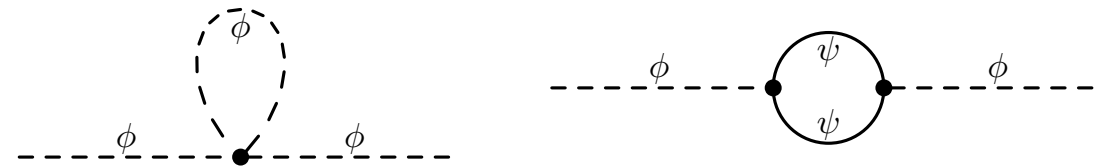

Figure 17: The divergent Feynman diagrams for the full $\mathcal{N}=1$ Wess-Zumino model in the $\mathbb{Z}_{2}$ symmetric phase.

with $A_{\hat{\mu}^{2}}$ defined for the SLAC derivative in Eq. (54). In contrast to the quenched model only a factor ' 1 ' in front of the divergent part is needed that arises from a partial cancellation of the Feynman diagrams shown in Fig. 17. Compared to the $\mathcal{N}=2$ Wess-Zumino model there is no complete cancellation and a divergence remains. Again the given renormalisation procedure amounts to a normal ordering of interaction terms with mass parameter $\hat{\mu}$ in the $\mathbb{Z}_{2}$ symmetric phase. Eventually a renormalised coupling is defined in the continuum limit similarly as in the bosonic case according to Eq. (50), but now with $\hat{\mu}_{0}^{2}$ given through Eq. (58).

\subsubsection{The Pfaffian}

The field content of the $\mathcal{N}=1$ Wess-Zumino model includes Majorana fermions for which the fermionic path integral yields a Pfaffian,

$$
\mathcal{Z}=\int \mathrm{D} \phi \mathrm{D} \psi \mathrm{e}^{-S_{\mathrm{B}}[\psi]-\psi^{\top} M[\phi] \psi}=\int \mathrm{D} \phi \operatorname{Pf} M[\phi] \mathrm{e}^{-S_{\mathrm{B}}[\phi]} .
$$

where $M[\phi]$ is an antisymmetric matrix ${ }^{23}$ The Pfaffian is (up to a sign) the square root of the determinant, $(\operatorname{Pf} M)^{2}=$ $\operatorname{det} M$, and follows from Grassmann integration,

$$
\text { Pf } M=\frac{1}{2^{N} N !} \sum_{\sigma \in S_{2 N}} \operatorname{sign}(\sigma) \prod_{i=1}^{N} M_{\sigma_{2 i-1}, \sigma_{2 i}} .
$$

In practice the Pfaffian is computed as described in [87, 88] with complexity $\mathcal{O}\left(N^{3}\right)$. Similar to the case of Dirac fermions in Sec. 2.2.1 this Pfaffian may have a fluctuating sign and Monte-Carlo simulations are carried out with the effective action

$$
S_{\text {eff }}=S_{\mathrm{B}}-\ln |\operatorname{Pf} M|=S_{\mathrm{B}}-\frac{1}{2} \ln \operatorname{det} M \quad \Rightarrow \quad \mathcal{Z}=\int \mathrm{D} \phi \mathrm{e}^{-S_{\text {eff }}[\phi]} .
$$

\footnotetext{
${ }^{23}$ In general, as it is the case e.g. for supersymmetric Yang-Mills theories [86], the matrix $M$ is not necessarily real. However, it is still antisymmetric but does not need to be anti-Hermitian.
} 
Nevertheless, the sign of the Pfaffian must be taken into account by reweighting of measurements.

With the chosen representation of the Clifford algebra the fermion matrix is given by

$$
M=\left(\begin{array}{cc}
\partial_{1}^{\text {SLAC }} & \partial_{0}^{\text {SLAC }}-P^{\prime} \\
\partial_{0}^{\text {SLAC }}+P^{\prime} & -\partial_{1}^{\text {SLAC }}
\end{array}\right) .
$$

By using general identities for the Pfaffian of an antisymmetric matrix $A \in \mathbb{R}^{2 n \times 2 n}$ and general matrix $B \in \mathbb{R}^{2 n \times 2 n}$,

$$
\operatorname{Pf}(A)=(-)^{n} \operatorname{Pf}\left(A^{\top}\right), \quad \operatorname{Pf}\left(B A B^{\top}\right)=\operatorname{det}(B) \operatorname{Pf}(A),
$$

transformation properties under the $\mathbb{Z}_{2}$ symmetry $\phi \rightarrow-\phi$ of the bosonic potential $P^{2} / 2$ can be derived. For any lattice-derivative with antisymmetric matrix representation (e.g. for the SLAC derivative) on a lattice with $N$ points

$$
\begin{aligned}
& \operatorname{Pf}\left(\begin{array}{cc}
\partial_{1} & \partial_{0}-P^{\prime} \\
\partial_{0}+P^{\prime} & -\partial_{1}
\end{array}\right) \stackrel{\text { transpose }}{=}(-)^{N} \operatorname{Pf}\left(\begin{array}{cc}
-\partial_{1} & -\partial_{0}+P^{\prime} \\
-\partial_{0}-P^{\prime} & \partial_{1}
\end{array}\right) \\
&\left(\begin{array}{cc}
0 & -1 \\
1 & 0
\end{array}\right) \\
&={ }^{N} \operatorname{Pf}\left(\begin{array}{cc}
\partial_{1} & \partial_{0}+P^{\prime} \\
\partial_{0}-P^{\prime} & -\partial_{1}
\end{array}\right)
\end{aligned}
$$

holds true. Under the $\mathbb{Z}_{2}$ transformation $P^{\prime} \rightarrow-P^{\prime}$ the Pfaffian is invariant on even lattice volumes and hence it defines a discrete symmetry of the full model. On the other hand, for an odd number of lattice sites the Pfaffian changes sign under $P^{\prime} \rightarrow-P^{\prime}$. The seemingly inconsistent behaviour where symmetry properties depend on the number of lattice points can be resolved by a closer look at the SLAC derivative. For this derivative the number of lattice points is directly related to the boundary conditions of the fields. By imposing the natural condition that the spectrum of the lattice derivative operator lies symmetric around the real axis in momentum space an even number of lattice points must be used for antiperiodic boundary conditions and an odd number for periodic ones 24 In consequence the Pfaffian changes sign under $P^{\prime} \rightarrow-P^{\prime}$ for periodic boundary conditions. Then the contribution of every field configuration with positive Pfaffian is cancelled in the path integral by the contribution of the transformed configuration with negative Pfaffian and the same bosonic action. This implies a vanishing Witten index, which is nothing but the path integral with periodic fermionic boundary conditions. For antiperiodic fermions in the temporal direction the Pfaffian keeps its sign under the $\mathbb{Z}_{2}$ symmetry in accordance with the positive definite partition function for the thermal ensemble.

For Wilson fermions the situation is different. In that case the derivative has still an antisymmetric matrix representation but the contribution of the Wilson term leads to

$$
\begin{aligned}
& \operatorname{Pf}\left(\begin{array}{cc}
\partial_{1} & \partial_{0}-P^{\prime}-\frac{r}{2} \Delta \\
\partial_{0}+P^{\prime}+\frac{r}{2} \Delta & -\partial_{1}
\end{array}\right) \stackrel{\text { transp. }}{=}(-)^{N} \operatorname{Pf}\left(\begin{array}{cc}
-\partial_{1} & -\partial_{0}+P^{\prime}+\frac{r}{2} \Delta \\
-\partial_{0}-P^{\prime}-\frac{r}{2} \Delta & \partial_{1}
\end{array}\right) \\
&\left(\begin{array}{cc}
0 & -1 \\
1 & 0
\end{array}\right) \\
&=(-)^{N} \operatorname{Pf}\left(\begin{array}{cc}
\partial_{1} & \partial_{0}+P^{\prime}+\frac{r}{2} \Delta \\
\partial_{0}-P^{\prime}-\frac{r}{2} \Delta & -\partial_{1}
\end{array}\right) .
\end{aligned}
$$

Changing the sign of $P^{\prime}$ can in general only preserve the modulus of the Pfaffian if the Wilson parameter $r$ changes its sign, too. Since the lattice theory is defined with fixed $r$ the $\mathbb{Z}_{2}$ symmetry will be broken by the Wilson term, similar to the $\mathcal{N}=2$ Wess-Zumino model [60]. For that reason the discretisation based on the SLAC derivative extends the works [37, 80, 89] by implementing the $\mathbb{Z}_{2}$ symmetry of the continuum model explicitly on the lattice.

\subsubsection{Symmetries, boundary conditions, and ground states}

The static properties under the $\mathbb{Z}_{2}$ symmetry that depend on the boundary conditions for the dynamical fermions can be directly related to the ground state structure of the full model. In the case of broken $\mathbb{Z}_{2}$ symmetry one ground state is correlated to positive $\tilde{\phi}$ while the other one is correlated to negative $\tilde{\phi}$. The analysis of $\operatorname{sign} \operatorname{Pf} M$ reveals that in exactly one of these states the sign flips when one changes the boundary conditions. A change of the fermionic boundary conditions amounts to an insertion of $(-1)^{N_{\mathrm{F}}}$ into the path integral which exactly implies the existence of one bosonic and one fermionic ground state.

\footnotetext{
${ }^{24}$ Nevertheless, the squared SLAC derivative can be defined unambiguously for periodic fields with an even number of lattice points.
} 

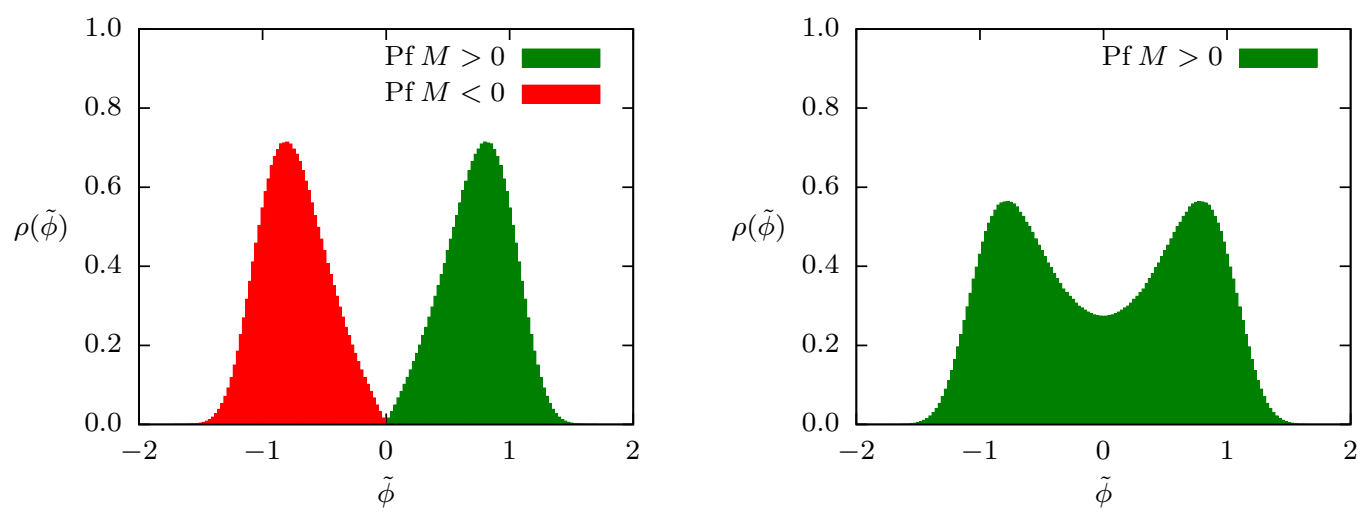

Figure 18: Probability density of the volume averaged bosonic field for periodic boundary conditions on a $9 \times 9$ lattice (left panel) and thermal boundary conditions on an $8 \times 9$ lattice (right panel) at coupling $\hat{f}=100$ and $\hat{\lambda}=0.1$. The histograms are computed (with a statistics of $6 \cdot 10^{6}$ configurations) separately for fixed sign $\operatorname{Pf} M$.

These relations have been checked in the dynamical ensemble with (small scale) lattice simulations with periodic and antiperiodic temporal boundary conditions for the fermions at couplings $\hat{\lambda}=0.1$ and $\hat{f}=100$ (see Fig. 18). The different boundary conditions at finite temperature imply that the functional forms of both histograms need not necessarily coincide. However, all configurations with $\tilde{\phi}>0$ keep $\operatorname{sign} \operatorname{Pf} M$ under a change of fermionic boundary conditions, such that the bosonic ground state is located at $\tilde{\phi}>0$, while the fermionic has a support $\tilde{\phi}<0$. These relations have been checked at further couplings $\hat{f} \in[10,100]$ and $\operatorname{sign} \tilde{\phi} \cdot \operatorname{sign} \operatorname{Pf} M>0$ is found on every configuration. Similar to the broken supersymmetric quantum mechanics in Sec. 2 it is necessary to use thermal boundary conditions to have a positive measure 25

This choice of thermal boundary conditions has further implications for the analysis of supersymmetry breaking. Apart from the explicit supersymmetry breaking introduced in the lattice theory by the finite lattice spacing and finite volume there is a further explicit breaking introduced by the finite temperature. For that reason an analysis of the spontaneous supersymmetry breaking in the continuum theory will involve the limit of infinite volume ("thermodynamic limit"), vanishing temperature, and vanishing lattice spacing of the lattice theory.

\subsection{4. $\mathbb{Z}_{2}$ breaking}

The numerical survey of the phase diagram starts in analogy to the quenched case with the determination of the phase transition associated to the $\mathbb{Z}_{2}$ breaking. The binder cumulant $U$ is thus computed for different volumes at fixed lattice spacing with thermal boundary conditions. However, a comparison to results obtained with periodic boundary conditions (without reweighting) is enlightening. For $\hat{\lambda}=0.3$ the intersection point of the Binder cumulants is independent of the chosen boundary conditions (see Fig. 19). This behaviour is explained by the ground state structure at infinite lattice volume. In the $\mathbb{Z}_{2}$ broken phase (for small $\hat{\mu}^{2}$ ) the system resides in only one ground state with fixed $\operatorname{sign} \operatorname{Pf} M$. In that case expectation values are insensitive to the boundary conditions for the fermions and periodic boundary may be imposed. For that reason it is quite safe to approach the phase transition from the $\mathbb{Z}_{2}$ broken region and extract a critical coupling from a crossing of Binder cumulants at the edge of the $\mathbb{Z}_{2}$ broken phase. But in the $\mathbb{Z}_{2}$ symmetric phase reweighted expectation values are undefined for periodic boundary conditions and un-reweighted values may only be used with caution.

The phase transition has also been determined for two further lattice spacings with thermal boundary conditions (see Fig. 20) and the results for every $\hat{\lambda}$ are in full agreement with a critical coupling of $f_{\mathrm{c}}=21.1(1.1)$. At this point the numerical precision is just not sufficient to resolve any running of the critical coupling with varying lattice spacing, mainly because of the accessible lattice sizes. Hence the determined critical couplings are used as continuum critical coupling for the $\mathbb{Z}_{2}$ breaking with a broken phase for $f>f_{\mathrm{c}}$ and a symmetric phase for $f<f_{\mathrm{c}}$.

\footnotetext{
${ }^{25}$ The Pfaffian is found to be strictly positive for finite temperature simulations with several couplings on lattices up to $20 \times 21$. However, for even larger lattices the calculation of the Pfaffian is getting extremely time consuming and a representative statistics has not been generated.
} 

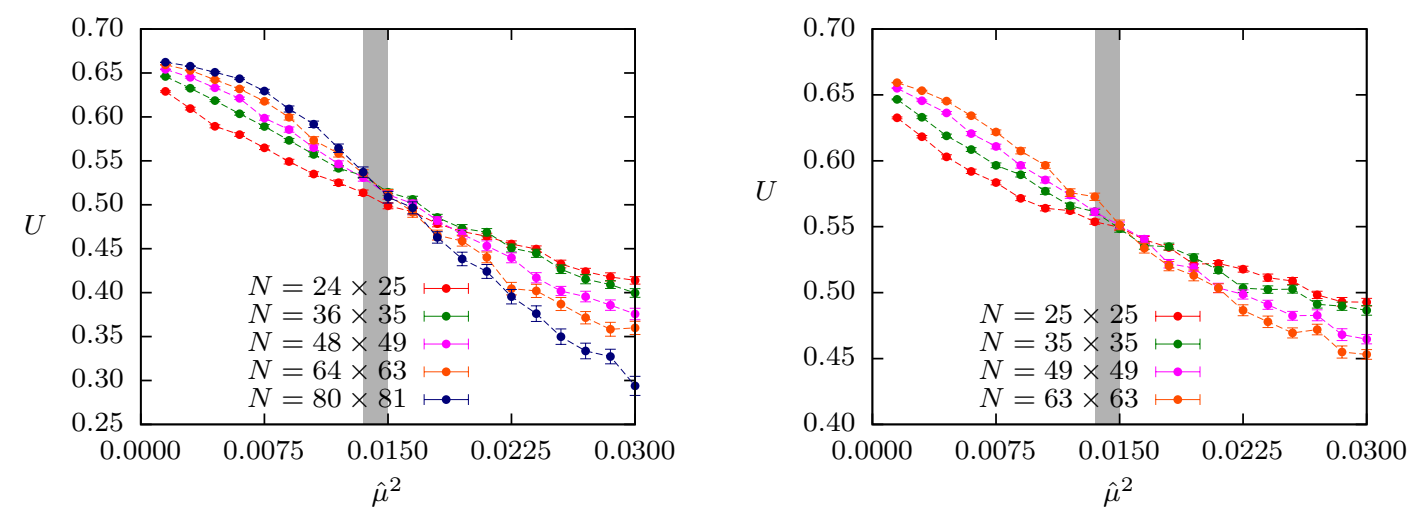

Figure 19: Binder cumulants for thermal (left panel) and supersymmetry preserving (right panel) boundary conditions determined at fixed $\hat{\lambda}=0.3$ and varying $\hat{\mu}^{2}$ (with a statistic of $10^{4}$ configurations). The shaded area denotes the error bounds of the critical $\hat{\mu}^{2}$.
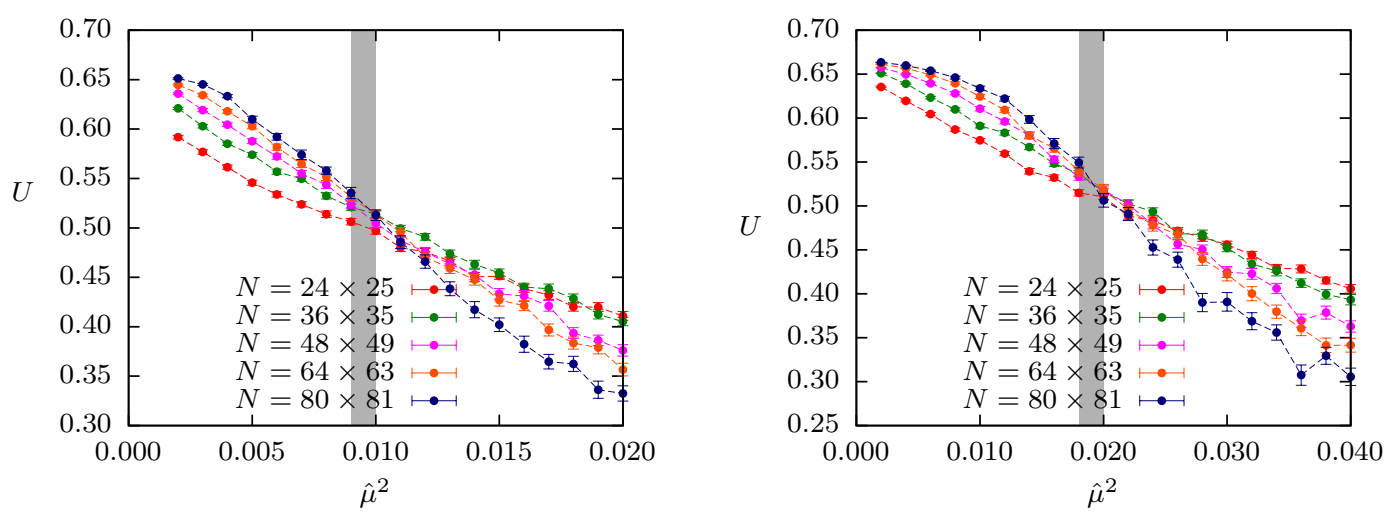

Figure 20: Binder cumulants for thermal boundary conditions determined at fixed $\hat{\lambda}=0.2$ (left panel) and $\hat{\lambda}=0.4$ (right panel) with a critical $\hat{\mu}^{2}$ indicated by the shaded area.

\subsubsection{Supersymmetry breaking}

As found in Sec. 3.2 .3 the $\mathcal{N}=1$ Wess-Zumino model with the chosen prepotential possesses one bosonic and one fermionic ground state which are related through the $\mathbb{Z}_{2}$ symmetry. In the $\mathbb{Z}_{2}$ broken phase one definite ground state is selected and this ground state will be the supersymmetric one because its partner state is not present in the physical spectrum at infinite volume. On the other hand, in the $\mathbb{Z}_{2}$ symmetric phase there may exist a supersymmetric ground state - in contrast to supersymmetric quantum mechanics does the $\mathbb{Z}_{2}$ symmetry not necessarily exclude a supersymmetric ground state. Thus it is necessary to study supersymmetry breaking on its own.

The direct way to study supersymmetry breaking is given by Ward identities that are related to the supersymmetry transformation. If there is one broken Ward identity then supersymmetry is broken. The simplest Ward identity which is inherently related to the ground state energy is constructed from the transformation of the fermionic field,

$$
\begin{aligned}
-\left\langle V^{-1} \int \mathrm{d}^{2} x \delta \psi\right\rangle=\epsilon\left\langle V^{-1} \int \mathrm{d}^{2} x P\right\rangle & =0 \quad \Leftrightarrow \quad\langle\mathcal{P}\rangle=0 \\
& \text { with }\langle\mathcal{P}\rangle=\langle\hat{\mathcal{P}}\rangle / \sqrt{\hat{\lambda}}, \quad \hat{\mathcal{P}}=N^{-1} \sum_{x}\left(\frac{\hat{\mu}_{0}^{2}}{\sqrt{2 \hat{\lambda}}}+\sqrt{\frac{\hat{\lambda}}{2}} \phi^{2}\right) .
\end{aligned}
$$

It follows that the dimensionless prepotential $\mathcal{P}$ serves as an indicator for supersymmetry breaking. However, supersymmetry is explicitly broken by the finite lattice spacing, finite temperature and finite volume. Therefore dynamical supersymmetry breaking should be examined in the limit of infinite lattice volume $N \rightarrow \infty$, in combination with the continuum limit $a \rightarrow 0$. 

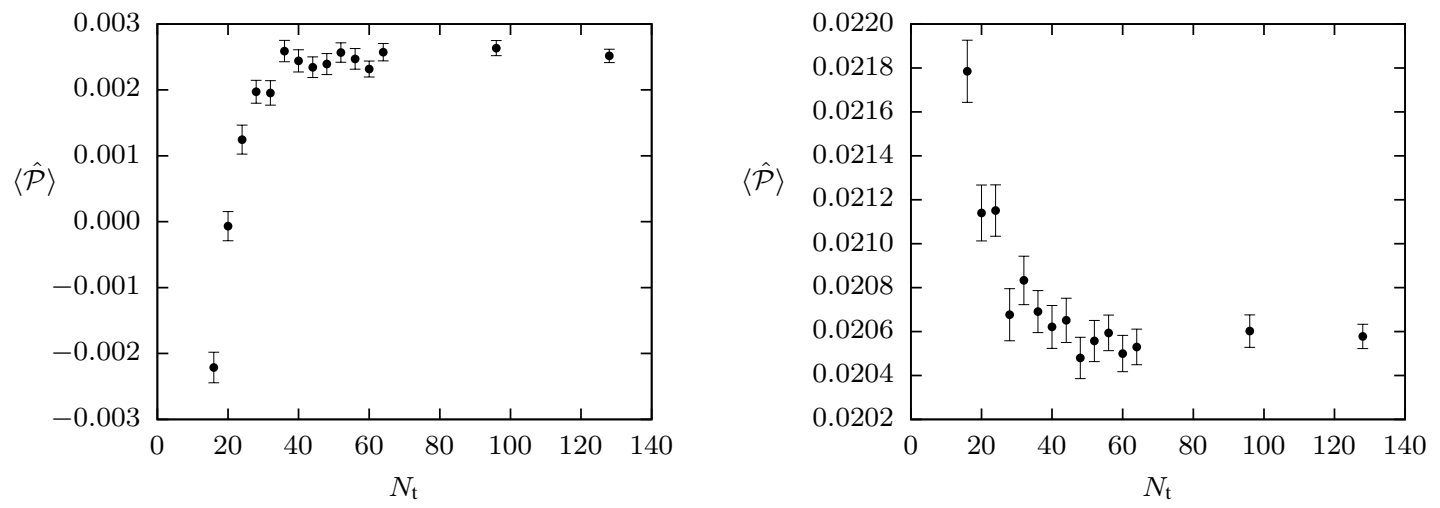

Figure 21: Prepotential $\langle\hat{\mathcal{P}}\rangle$ at fixed $\hat{\lambda}=0.1$ and $N_{\mathrm{s}}=35$ for the $\mathbb{Z}_{2}$ broken phase with $\hat{f}=100$ (left panel) and the $\mathbb{Z}_{2}$ symmetric phase with $f=10$ (right panel).
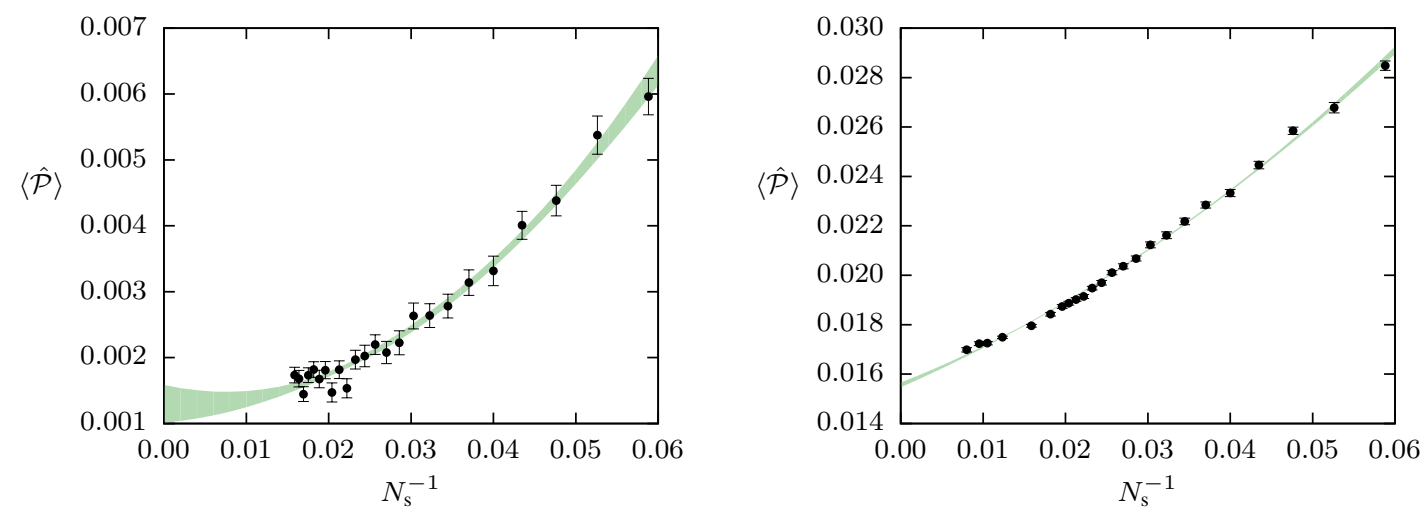

Figure 22: Extrapolation of $\langle\hat{\mathcal{P}}\rangle$ to infinite volume at fixed $\hat{\lambda}$ and couplings $\hat{f}=100$ (left panel) and $\hat{f}=10$ (right panel). Depending on the lattice size from $10^{4}$ up to $5 \cdot 10^{5}$ configurations have been used.

The first limit to be studied is the limit of vanishing temperature at fixed lattice spacing $(\hat{\lambda}=0.1)$ and fixed spatial volume $\left(N_{\mathrm{s}}=35\right)$. Simulations have been performed at $\hat{f}=100\left(\mathbb{Z}_{2}\right.$ unbroken $)$ and $\hat{f}=10\left(\mathbb{Z}_{2}\right.$ broken $)$ and the results plotted in Fig. 21 indicate that for $N_{\mathrm{t}} \gtrsim N_{\mathrm{s}}$ the finite temperature corrections become negligible in both phases. Hence in the following nearly quadratic lattices are chosen with $N_{\mathrm{t}}=N_{\mathrm{s}} \pm 126$ But already at this point the prepotential is one order of magnitude larger in the $\mathbb{Z}_{2}$ symmetric phase than in the $\mathbb{Z}_{2}$ broken phase.

Now that the finite temperature effects are under control the infinite volume limit can be performed. Here, the infinite volume limit is taken before the continuum limit to finally work out the effect of the finite lattice spacing. To accomplish an extrapolation the data at fixed finite lattice spacing are fitted with functions of the form

$$
\langle\hat{\mathcal{P}}\rangle\left(N_{\mathrm{s}}\right)=A+B N_{\mathrm{s}}^{-1}+C N_{\mathrm{s}}^{-2}
$$

and extrapolated to $N_{\mathrm{s}} \rightarrow \infty$. For most of the couplings $\hat{f}$ lattices with $N_{\mathrm{s}} \in\{25,27,31,35,43,63\}$ are used. The two examples with $\hat{\lambda}=0.1$ and $\hat{f} \in\{10,100\}$ in Fig. 22 illustrate the validity of the chosen extrapolation formula.

The last limit to be taken is the continuum limit. In the simplest case corrections are of $\mathcal{O}(a)$ and a linear extrapolation to the continuum limit is possible. The extrapolation is done at each coupling $f \in\{10,12.5,16,20,25,40,100\}$ and is shown for the extreme cases of the present study, at $f=10$ and $f=100$, in Fig. 23. where the validity of a linear extrapolation is visible. In these cases a complete coincidence between restored $\mathbb{Z}_{2}$ symmetry and spontaneously broken supersymmetry is visible. All continuum extrapolated Ward identities in the considered coupling range are plotted in Fig. 24 and listed in Tab. 3.

\footnotetext{
${ }^{26}$ The choice of the sign in $N_{\mathrm{s}} \pm 1$ depends on the efficiency of the involved Fourier transformations.
} 

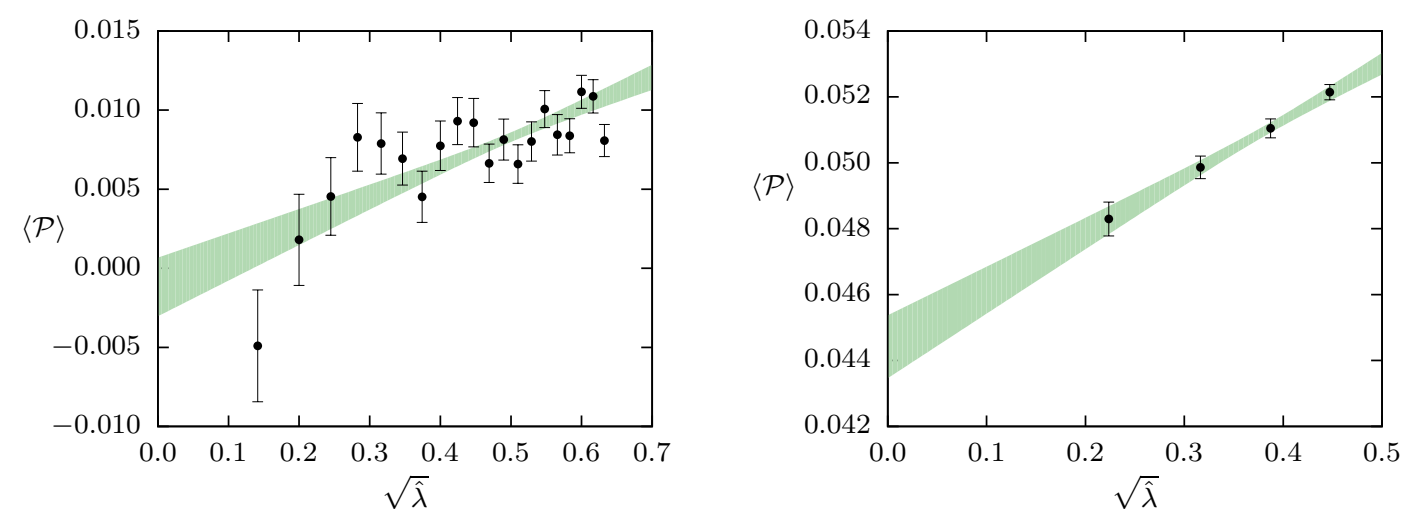

Figure 23: For the $\mathbb{Z}_{2}$ broken case $\left(f=100\right.$, left panel) the Ward identity is fulfilled with $\langle\mathcal{P}\rangle=-0.0012(19)$. For restored $\mathbb{Z}_{2}$ symmetry at $f=10$ (right panel) $\langle\mathcal{P}\rangle=0.0444(10)$ is obtained.

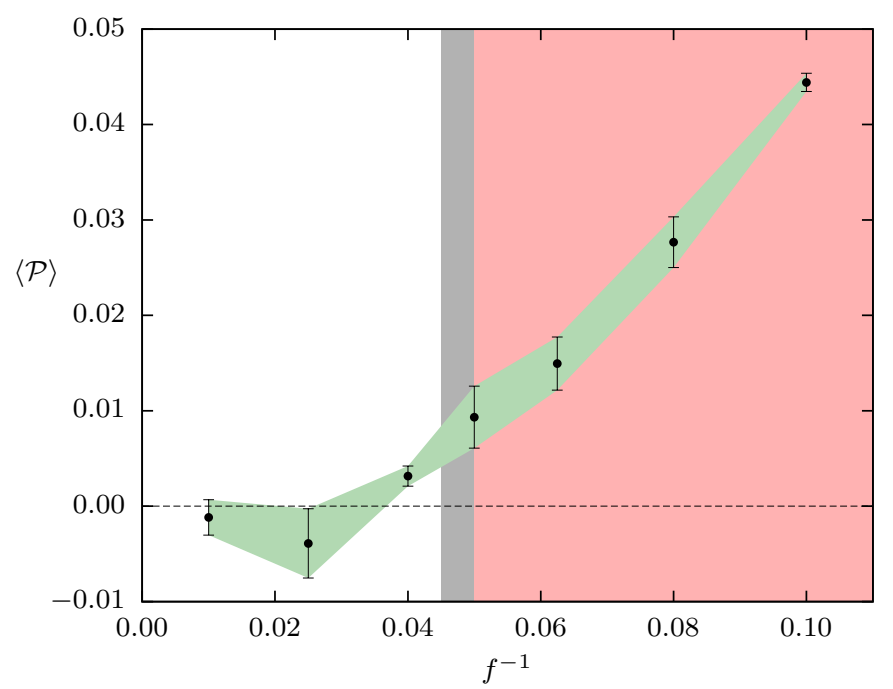

Figure 24: Dimensionless prepotential $\mathcal{P}$ over inverse coupling $f^{-1}$. The region shaded in red indicates the $\mathbb{Z}_{2}$ restored coupling range where supersymmetry is broken while the region shaded in gray gives the error bound for the $\mathbb{Z}_{2}$ phase transition.

The calculation of the dimensionless prepotential that serves as Ward identity by Eq. 66 has shown that after taking all necessary limiting procedures supersymmetry is broken whenever the $\mathbb{Z}_{2}$ symmetry is restored. Nevertheless, one inconsistency shows up for $f$ slightly above the critical coupling for $\mathbb{Z}_{2}$ breaking (approximately $f \in\left[f_{\mathrm{c}}, 27\right]$ ). In that region the $\mathbb{Z}_{2}$ symmetry is broken while the Ward identity is not fulfilled. This is in contradiction with the fact that a broken $\mathbb{Z}_{2}$ symmetry strictly implies a restored supersymmetry after all limits have been taken. Thus an analysis of possible systematic errors is in order.

Firstly the used extrapolation formulae for the specific limits may not be sufficient in every case. Secondly close to the critical coupling at fixed $\hat{\lambda}$ where a second order phase transition (related to an infinite continuum $\lambda$ ) with diverging correlation length occurs, the considered lattice volumes may still be too small to be in the applicability range for an infinite volume extrapolation with Eq. (67). Thirdly the ordering of limits may be of importance. As it has been found in the supersymmetric quantum mechanics and the $\mathcal{N}=2$ Wess-Zumino model the sign problem that arises from the fermionic part of the action becomes worse for larger volumes at fixed lattice spacing and is weakened in the continuum limit at fixed physical volume. Although the sign problem is completely absent for small lattices sign Pf $M$ has not been computed for the larger lattices due to the numerical complexity of $\mathcal{O}\left(N^{3}\right)$. Therefore sign problems cannot be excluded for large lattice volumes and an extrapolation to infinite volume prior to the continuum limit may turn out to be insufficient if no reweighting with $\operatorname{sign} \operatorname{Pf} M$ is done. 


\begin{tabular}{cc}
\hline$f$ & $\langle\mathcal{P}\rangle$ \\
\hline 100 & $-0.0012(19)$ \\
40 & $-0.0039(36)$ \\
25 & $0.0032(11)$ \\
20 & $0.0093(33)$ \\
16 & $0.0150(28)$ \\
12.5 & $0.0277(27)$ \\
10 & $0.0444(10)$ \\
\hline
\end{tabular}

Table 3: Dimensionless prepotential $\mathcal{P}$ after extrapolation to the limit of vanishing temperature, infinite volume, and vanishing lattice spacing (in given order).

\begin{tabular}{llcccc}
\hline & & $N_{\mathrm{s}}=49$ & $N_{\mathrm{s}}=63$ & $N_{\mathrm{s}}=81$ & cont. \\
\hline$L \sqrt{\lambda}=19.9$ & bos. & $0.379(2)$ & $0.379(4)$ & $0.383(4)$ & $0.389(10)$ \\
$L \sqrt{\lambda}=28.2$ & bos. & $0.295(4)$ & $0.293(5)$ & $0.293(5)$ & $0.289(12)$ \\
$L \sqrt{\lambda}=34.5$ & bos. & $0.267(6)$ & $0.262(8)$ & $0.261(7)$ & $0.251(20)$ \\
$L \sqrt{\lambda}=39.8$ & bos. & $0.238(10)$ & $0.228(12)$ & $0.231(10)$ & $0.218(30)$ \\
$L \sqrt{\lambda}=19.9$ & ferm. & $0.300(1)$ & $0.294(2)$ & $0.291(2)$ & $0.277(4)$ \\
$L \sqrt{\lambda}=28.2$ & ferm. & $0.276(1)$ & $0.263(2)$ & $0.262(2)$ & $0.237(5)$ \\
$L \sqrt{\lambda}=34.5$ & ferm. & $0.270(1)$ & $0.261(2)$ & $0.252(2)$ & $0.225(5)$ \\
$L \sqrt{\lambda}=39.8$ & ferm. & $0.270(1)$ & $0.254(3)$ & $0.245(2)$ & $0.204(6)$ \\
\hline
\end{tabular}

Table 4: Bosonic (upper rows) and fermionic (lower rows) masses $m_{\mathrm{B} / \mathrm{F}} / \sqrt{\lambda}$ for fixed physical volumes $L \sqrt{\lambda}$ and varying lattice spacing together with the continuum extrapolation at coupling $f=100$.

Even with taking these possible systematic errors into account a supersymmetry breaking phase transition is confirmed where the corresponding critical coupling coincides with that of the $\mathbb{Z}_{2}$ phase transition. Nevertheless, the Binder cumulant technique for the $\mathbb{Z}_{2}$ symmetry breaking provides a more reliable way to determine the critical coupling because the extrapolation does not directly involve questionable extrapolation formulae.

\subsubsection{Masses}

Ward identities are indicators for the restoration of supersymmetry. Now, after the phase structure of the theory is settled, further physical observables are of interest. Amongst them are the particle masses or the energy difference between the ground state and the first excited state. Here, one expects a fundamentally different behaviour of the masses in the different phases [77]. In the supersymmetric phase a degeneracy between the (finite) bosonic and fermionic mass is expected, similar to the $\mathcal{N}=2$ Wess-Zumino model. For broken supersymmetry a goldstino should arise as massless fermionic mode while the physical spacetime volume serves as a regulator for the bosonic mass, which itself eventually vanishes in the infinite volume limit.

The analysis starts with the $\mathbb{Z}_{2}$ broken phase at a fixed coupling of $f=100$. In this phase it is necessary to project the (finite volume) lattice simulations onto one ground state to mimic the suppression of tunnelling events in the infinite volume limit 27 This is the same technique as used in Sec. 2 for the SQM as well as for the $\mathcal{N}=2$ Wess-Zumino model in [60], and it is necessary to finally extrapolate the obtained masses to the infinite volume limit. Since a projection to one ground state is performed it is not required to stick to thermal boundary conditions as discussed in Sec. 3.2.4 Thus, in order to remove the supersymmetry breaking introduced by a finite temperature, periodic boundary conditions are used also for the fermionic field to obtain correlators and masses in the $\mathbb{Z}_{2}$ broken phase. Furthermore only square lattices are investigated to simplify the comparison of different lattice spacings and physical volumes.

\footnotetext{
${ }^{27}$ Here the configurations are projected without loss of generality to the bosonic ground state.
} 

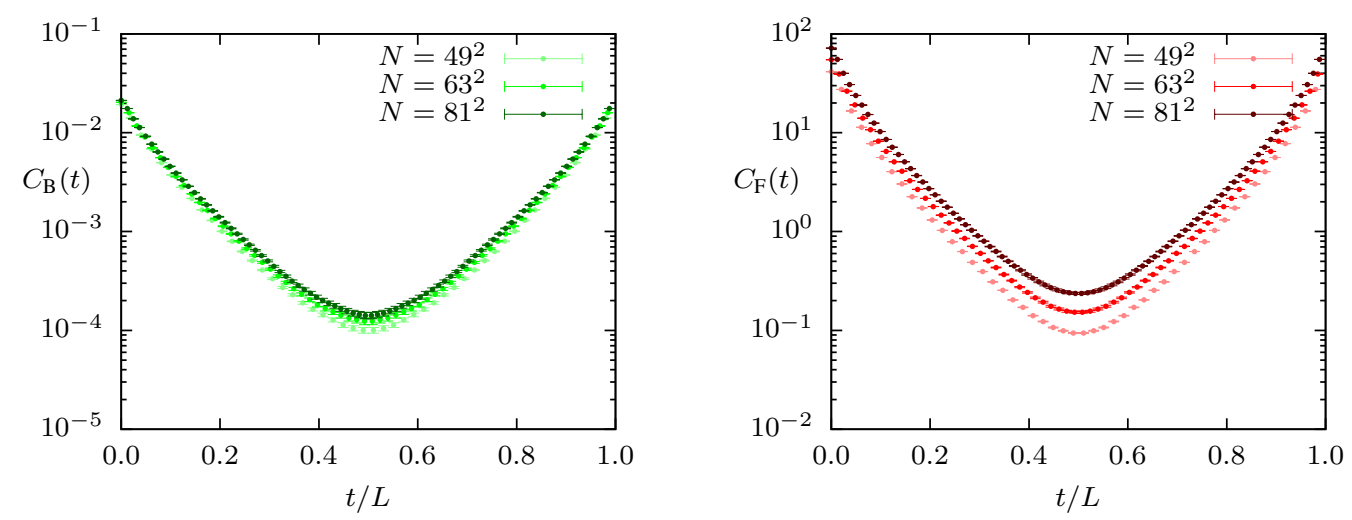

Figure 25: Connected bosonic (left panel) and fermionic (right panel) correlator in the $\mathbb{Z}_{2}$ broken phase $(f=100)$ at fixed physical volume $L \sqrt{\lambda}=39.8$ for different lattice spacings.
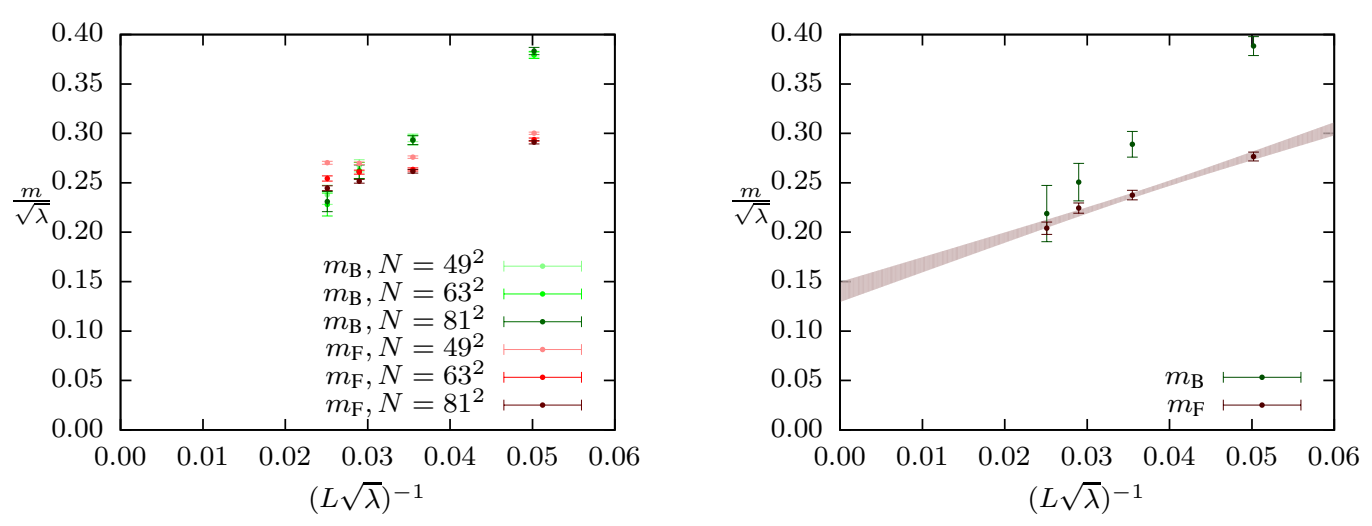

Figure 26: Left panel: Masses at finite lattice spacing at coupling $f=100$. Right panel: Continuum extrapolated masses together with the error bounds of an extrapolation of the fermionic mass to the infinite volume limit (shaded area). $10^{6}$ configurations have been used for each data point.

Masses are extracted from the correlators

$$
C_{\mathrm{B}}(t)=N_{\mathrm{s}}^{-2} \sum_{x, x^{\prime}}\left\langle\phi_{(t, x)} \phi_{\left(0, x^{\prime}\right)}\right\rangle \quad \text { and } \quad C_{\mathrm{F}}(t)=N_{\mathrm{s}}^{-2} \sum_{\alpha, x, x^{\prime}}\left\langle\bar{\psi}_{\alpha,(t, x)} \psi_{\alpha,\left(0, x^{\prime}\right)}\right\rangle
$$

using a cosh fit in a range $t \in[L / 3,2 L / 3]$. It is obvious that the correlators at fixed physical volume depend on the lattice spacing (in Fig. 25 the fermionic correlator shows larger discretisation errors) and extracted masses must therefore be extrapolated to the continuum limit. The continuum value is reached via a linear extrapolation that has already been used successfully for the continuum extrapolation of results based on the SLAC derivative in unbroken supersymmetric quantum mechanics in [42]. The results obtained on lattices with $N_{\mathrm{s}} \in\{49,63,81\}$ for the bosonic and fermionic masses at four different physical volumes, together with the continuum extrapolation, are given in Tab. 4 and are plotted in Fig. 26 (left panel) for finite lattice spacings. Finally an infinite volume extrapolation of the continuum results is necessary. Although boson masses approach the fermion masses at larger volumes, as predicted by supersymmetry, the statistical accuracy is not sufficient for a reliable extrapolation. Therefore only the fermionic masses are extrapolated linearly (see Fig. 26, right panel) to the infinite volume limit, resulting in $m_{\mathrm{F}} / \sqrt{\lambda}=0.14(1)$. Unfortunately, the bosonic mass can only be assumed to take the same limit. At this point a note concerning the finiteness of the bosonic mass is in order. The breaking of the discrete $\mathbb{Z}_{2}$ symmetry does not lead to Goldstone bosons and a finite mass is not excluded. On the other hand, in the $\mathbb{Z}_{2}$ symmetric phase the continuous supersymmetry will be broken and massless goldstinos should appear in the spectrum of the theory.

The analysis of masses is therefore continued in the phase with broken supersymmetry and restored $\mathbb{Z}_{2}$ symmetry. In this phase it is mandatory to use thermal boundary conditions since bosonic and fermionic ground state participate 

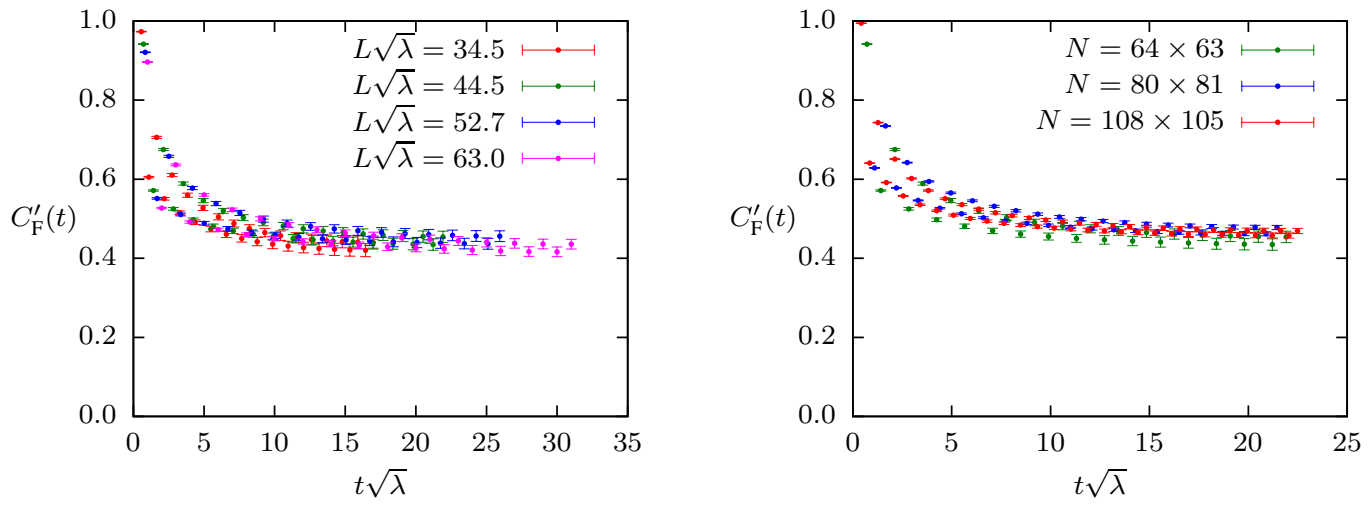

Figure 27: Fermionic correlators at coupling $f=10$ in the thermal ensemble for different physical volumes on a $64 \times 63$ lattice (left panel) and for different lattice sizes on a fixed physical volume $L \sqrt{\lambda}=44.5$ (right panel). For symmetry reasons only the $t / L<0.5$ range is shown.

equally well in the path integral with unsuppressed tunnelling even at infinite volume. Goldstinos will, similar to broken supersymmetric quantum mechanics, show up as massless modes in

$$
C_{\mathrm{F}}^{\prime}(t)=N_{\mathrm{s}}^{-2} \sum_{\alpha, x, x^{\prime}}\left\langle\bar{\psi}_{1,(t, x)} \psi_{1,\left(0, x^{\prime}\right)}-\bar{\psi}_{2,(t, x)} \psi_{2,\left(0, x^{\prime}\right)}\right\rangle
$$

the spinor component combination that yields a cosh form for thermal boundary conditions. At $\hat{f}=10$ the correlator has been computed at varying physical volume with fixed lattice size and for fixed physical volume with varying lattice spacing (see Fig. 27) 28 A constant part of the correlator is clearly visible and independent of the physical volume or lattice spacing. This is an unambiguous sign of a goldstino.

To get a complete picture the bosonic masses are calculated as well. These are expected to vanish in the infinite volume limit as predicted by renormalisation group methods in [77]. Again, the connected bosonic correlator at $f=10$ is computed and it is found to be composed of a part with nearly vanishing mass, corresponding to the first excited state, and a part that arises from higher excited states (see Fig. 28, left panel) 29 The masses of the first excited state are now extrapolated (linearly) to infinite volume (see Fig. 28, right panel). Here, only constant lattice sizes $N=64 \times 63$ are used and the continuum limit is not performed. However, it has been checked for $L \sqrt{\lambda}=44.5$ on lattice sizes $N=80 \times 81$ and $N=108 \times 105$ that the discretisation errors are still below the statistical errors. The infinite volume extrapolation is in agreement with $m_{\mathrm{B}} \propto L^{-1}$ with an extrapolated value of $m_{\mathrm{B}} / \sqrt{\lambda}=-0.002(10)$, i.e. the bosonic mass vanishes after the infrared regulator is removed, in agreement with the results obtained by a functional renormalisation group approach [77].

\section{Conclusions}

For the case of a supersymmetric quantum mechanics with dynamically broken supersymmetry observables that are computed using a lattice regularisation with the SLAC derivative completely coincide with results obtained from the diagonalised Hamiltonian. The (bosonic/fermionic) nature of both ground states can be explained with the impact of a change in boundary conditions on the fermionic determinant. Correlators computed in the thermal ensemble show a constant part for large $t$, which is a remnant of the degenerate ground states. With a projection to one ground state the constant part is still visible in the fermionic correlator, which goes at hand with the massless fermionic excitation implied by the degeneracy. In the bosonic correlator the constant part vanishes and the remaining exponential fall-off corresponds to the first excited state in the bosonic spectrum.

\footnotetext{
${ }^{28}$ The non-statistical fluctuations showing up in the correlator can be traced back to the non-locality of the SLAC derivative. They will decrease in the continuum limit, as visible in Fig.27](right panel).

${ }^{29}$ The exponential decay of higher excited states is also visible for $t \sqrt{\lambda}<10$ in the fermionic correlator, cf. Fig.27
} 

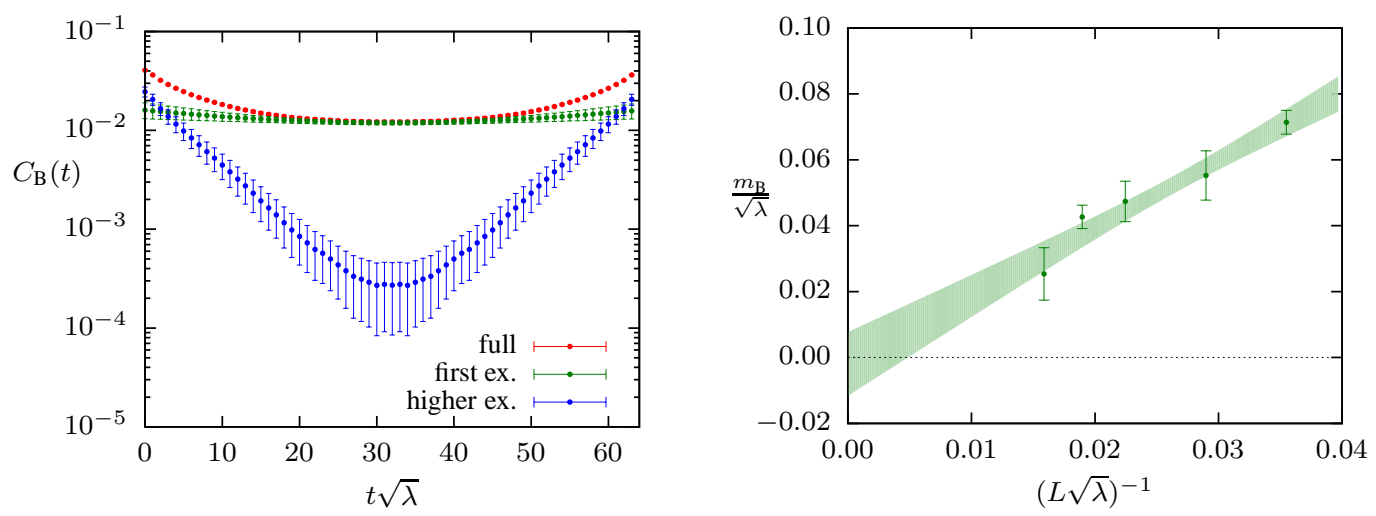

Figure 28: Left panel: Bosonic correlator at couping $f=10$ on a $64 \times 63$ lattice for fixed physical volume $L \sqrt{\lambda}=63$ where the contributions of first and higher excited states have been separated. Right panel: Bosonic mass of the first excited state on $64 \times 63$ lattices and the infinite volume extrapolation (shaded area) for $f=10$.

On the level of Ward identities it is checked that the ground state is not invariant under the supersymmetry and a simple Ward identity is not fulfilled in the limit of vanishing lattice spacing and temperature, as predicted from diagonalising the Hamiltonian. In contrast to purely bosonic scalar models, the constraint effective potential does not flatten out towards the conventional effective potential, which is explained by the fact that no interpolating states between bosonic and fermionic sector are accessibly in the finite temperature path integral.

We showed that it is possible to analyse supersymmetric quantum mechanics with broken supersymmetry with lattice methods based on the SLAC derivative. The physical properties can be determined reliably and accurately. Supersymmetric quantum mechanics has been very useful to test sophisticated techniques that find applications in higher dimensional models.

The analysis of the $\mathcal{N}=(1,1)$ Wess-Zumino model in two dimensions aimed at observing and understanding dynamical supersymmetry breaking from first principles. A lattice regularisation based on the SLAC derivative is used and the choice of this regularisation is justified utilising the quenched model where a complete agreement of the obtained critical coupling with the reference value [83] is found.

With this discretisation the $\mathbb{Z}_{2}$ symmetry breaking is analysed and a renormalised continuum coupling is defined. For the first time a regulator independent critical coupling is determined from lattice simulations. From the computation of a Ward identity a complete coincidence between the restoration of $\mathbb{Z}_{2}$ symmetry and the dynamical breaking of supersymmetry is obtained. The computation of masses in the continuum limit for different physical volumes completes the analysis and agrees with the picture of a finite and equal bosonic and fermionic mass in the supersymmetric phase and the occurrence of a massless goldstino for broken supersymmetry.

In future works it may be checked on the Ward identities by taking the infinite volume limit after the continuum limit has been carried out to suppress possible systematic errors arising from the sign problem. Masses of higher excited states could be within reach by using improved correlators. Finally a completely independent calculation with different discretisation is desirable. A formulation with Wilson fermions [37] is a natural choice, in particular because efficient lattice methods are already available [90]. However, in that case one must ensure that a spontaneous breaking of the $\mathbb{Z}_{2}$ symmetry is not influenced by the unavoidable explicit $\mathbb{Z}_{2}$ symmetry breaking induced by the Wilson mass term.

In both models the SLAC derivative has proven to be successfully applicable, which can be traced back to the absence of gauge fields in these models. Therefore it may be advantageous to consider the SLAC derivative as an interesting alternative to Ginsparg-Wilson fermions also in simulations of the four dimensional $\mathcal{N}=1$ Wess-Zumino model [91].

Supersymmetrically improved lattice actions inevitably include periodic boundary conditions for fermionic fields which lead to a severe sign problem in supersymmetric theories with a dynamically broken supersymmetry. Therefore the applicability of the improvement programme, where one keeps part of the supersymmetry intact, becomes questionable for these theories. It will be necessary to analyse if improved actions exist that give rise to a correct continuum limit without fine tuning even for thermal boundary conditions. 


\section{Acknowledgements}

Numerous discussions with Tobias Kästner, Georg Bergner, and Franziska Synatschke are gratefully acknowledged. CW thanks for the support by the Studienstiftung des deutschen Volkes. This work has been supported by the DFG Research Training Group "Quantum and Gravitational Fields" GRK 1523 and the DFG grant Wi 777/10-1. The simulations have been carried out at the Omega cluster of the TPI.

\section{References}

[1] S. L. Glashow, Partial Symmetries of Weak Interactions, Nucl. Phys. 22 (1961) 579.

[2] A. Salam and J. C. Ward, Electromagnetic and weak interactions, Phys. Lett. 13 (1964) 168.

[3] S. Weinberg, A Model of Leptons, Phys. Rev. Lett. 19 (1967) 1264.

[4] H. Fritzsch, M. Gell-Mann and H. Leutwyler, Advantages of the Color Octet Gluon Picture, Phys. Lett. B47 (1973) 365.

[5] K. Kodama et al. (DONUT), Observation of tau-neutrino interactions, Phys. Lett. B504 (2001) 218 |arXiv:hep-ex/0012035|.

[6] S. R. Coleman and J. Mandula, All possible symmetries of the S matrix, Phys. Rev. 159 (1967) 1251.

[7] R. Haag, J. T. Lopuszanski and M. Sohnius, All Possible Generators of Supersymmetries of the S Matrix, Nucl. Phys. B88 (1975) 257.

[8] J. Wess and B. Zumino, A lagrangian model invariant under supergauge transformations, Phys. Lett. B49 (1974) 52.

[9] M. F. Sohnius, Introducing Supersymmetry, Phys. Rept. 128 (1985) 39.

[10] S. P. Martin, A Supersymmetry Primer, arXiv:hep-ph/9709356

[11] J. Wess and J. Bagger, Supersymmetry and supergravity, Princeton, USA: Univ. Pr., 1992.

[12] S. Dimopoulos and H. Georgi, Softly Broken Supersymmetry and SU(5), Nucl. Phys. B193 (1981) 150.

[13] J. R. Ellis, J. S. Hagelin, D. V. Nanopoulos, K. A. Olive and M. Srednicki, Supersymmetric relics from the big bang, Nucl. Phys. B238 (1984) 453.

[14] G. Hiller and M. Schmaltz, Solving the strong CP problem with supersymmetry, Phys. Lett. B514 (2001) 263 [arXiv:hep-ph/0105254].

[15] E. Witten, Constraints on Supersymmetry Breaking, Nucl. Phys. B202 (1982) 253.

[16] L. O'Raifeartaigh, Spontaneous Symmetry Breaking for Chiral Scalar Superfields, Nucl. Phys. B96 (1975) 331.

[17] E. Witten, Dynamical breaking of supersymmetry, Nucl. Phys. B188 (1981) 513.

[18] M. Creutz, Monte Carlo Study of Quantized SU(2) Gauge Theory, Phys. Rev. D21 (1980) 2308.

[19] S. Dürr et al., Ab-Initio Determination of Light Hadron Masses, Science 322 (2008) 1224 [arXiv:0906.3599|.

[20] A. Feo, Predictions and recent results in SUSY on the lattice, Mod. Phys. Lett. A19 (2004) 2387 [arXiv:hep-lat/0410012].

[21] J. Giedt, Deconstruction and other approaches to supersymmetric lattice field theories, Int. J. Mod. Phys. A21 (2006) 3039 arXiv:hep-lat/0602007].

[22] T. Takimi, Relationship between various supersymmetric lattice models, JHEP 07 (2007) 010 [arXiv:0705.3831].

[23] P. H. Damgaard and S. Matsuura, Lattice supersymmetry: Equivalence between the link approach and orbifolding, JHEP 09 (2007) 097 arXiv:0708.4129].

[24] S. Catterall, From twisted supersymmetry to orbifold lattices, JHEP 01 (2008) 048 arXiv:0712.2532.

[25] J. W. Elliott, J. Giedt and G. D. Moore, Lattice four-dimensional N=4 SYM is practical, arXiv:0806.0013.

[26] J. Giedt, Progress in four-dimensional lattice supersymmetry, Int. J. Mod. Phys. A24 (2009) 4045 [arXiv:0903.2443].

[27] M. G. Endres, Dynamical simulation of N=1 supersymmetric Yang-Mills theory with domain wall fermions, Phys. Rev. D79 (2009) 094503 arXiv:0902.4267].

[28] K. Demmouche et al., Simulation of $4 d N=1$ supersymmetric Yang-Mills theory with Symanzik improved gauge action and stout smearing, arXiv:1003.2073

[29] K. G. Wilson, Quarks and Strings on a Lattice, New Phenomena In Subnuclear Physics. Part A. Proceedings of the First Half of the 1975 International School of Subnuclear Physics, Erice, Sicily, July 11 - August 1, 1975, ed. A. Zichichi, Plenum Press, New York, 1977, p. 69, CLNS-321.

[30] H. B. Nielsen and M. Ninomiya, Absence of Neutrinos on a Lattice. 1. Proof by Homotopy Theory, Nucl. Phys. B185 (1981) 20.

[31] H. B. Nielsen and M. Ninomiya, Absence of Neutrinos on a Lattice. 2. Intuitive Topological Proof, Nucl. Phys. B193 (1981) 173.

[32] H. B. Nielsen and M. Ninomiya, No go theorem for regularizing chiral fermions, Phys. Lett. B105 (1981) 219.

[33] P. H. Ginsparg and K. G. Wilson, A Remnant of Chiral Symmetry on the Lattice, Phys. Rev. D25 (1982) 2649.

[34] P. H. Dondi and H. Nicolai, Lattice supersymmetry, Nuovo Cim. A41 (1977) 1.

[35] J. Giedt and E. Poppitz, Lattice supersymmetry, superfields and renormalization, JHEP 09 (2004) 029 [arXiv:hep-th/0407135].

[36] I. Montvay, Tuning to N=2 supersymmetry in the SU(2) adjoint Higgs-Yukawa model, Nucl. Phys. B445 (1995) 399 |arXiv:hep-lat/9503009|.

[37] M. F. L. Golterman and D. N. Petcher, A local interactive lattice model with supersymmetry, Nucl. Phys. B319 (1989) 307.

[38] S. Catterall, D. B. Kaplan and M. Unsal, Exact lattice supersymmetry, Phys. Rept. 484 (2009) 71 [arXiv:0903.4881].

[39] G. Bergner, F. Bruckmann and J. M. Pawlowski, Generalising the Ginsparg-Wilson relation: Lattice Supersymmetry from Blocking Transformations, Phys. Rev. D79 (2009) 115007 |arXiv:0807.1110|.

[40] G. Bergner, Complete supersymmetry on the lattice and a No-Go theorem: A simulation with intact supersymmetries on the lattice, JHEP 01 (2010) 024 arXiv:0909.4791].

[41] A. D'Adda, I. Kanamori, N. Kawamoto and J. Saito, Species Doublers as Super Multiplets in Lattice Supersymmetry: Chiral Conditions of Wess-Zumino Model for $D=N=2$, arXiv:1107.1629

[42] G. Bergner, T. Kästner, S. Uhlmann and A. Wipf, Low-dimensional supersymmetric lattice models, Annals Phys. 323 (2008) 946 arXiv:0705.2212].

[43] M. Creutz and B. Freedman, A statistical approach to quantum mechanics, Ann. Phys. 132 (1981) 427. 
[44] S. Catterall and E. Gregory, A lattice path integral for supersymmetric quantum mechanics, Phys. Lett. B487 (2000) 349 arXiv:hep-lat/0006013].

[45] M. Beccaria, G. Curci and E. D’Ambrosio, Simulation of supersymmetric models with a local Nicolai map, Phys. Rev. D58 (1998) 065009 arXiv:hep-lat/9804010].

[46] T. Kästner, G. Bergner, S. Uhlmann, A. Wipf and C. Wozar, Supersymmetric lattice models in one and two dimensions, PoS LAT2007 (2007) 265 [arXiv:0709.0822].

[47] J. Giedt, R. Koniuk, E. Poppitz and T. Yavin, Less naive about supersymmetric lattice quantum mechanics, JHEP 12 (2004) 033 arXiv:hep-lat/0410041].

[48] I. Kanamori, H. Suzuki and F. Sugino, Euclidean lattice simulation for the dynamical supersymmetry breaking, Phys. Rev. D77 (2008) 091502 [arXiv:0711.2099].

[49] I. Kanamori, F. Sugino and H. Suzuki, Observing dynamical supersymmetry breaking with euclidean lattice simulations, arXiv:0711.2132

[50] F. Cooper, A. Khare and U. Sukhatme, Supersymmetry and quantum mechanics, Phys.Rept. 251 (1995) 267 [arXiv:hep-th/9405029].

[51] A. Wipf, Non-perturbative methods in supersymmetric theories, arXiv:hep-th/0504180

[52] A. Kirchberg, J. D. Lange and A. Wipf, From the Dirac operator to Wess-Zumino models on spatial lattices, Ann. Phys. 316 (2005) 357 arXiv:hep-th/0407207].

[53] S. D. Drell, M. Weinstein and S. Yankielowicz, Strong coupling field theories. 2. Fermions and gauge fields on a lattice, Phys. Rev. D14 (1976) 1627.

[54] L. H. Karsten and J. Smit, The vacuum polarization with SLAC lattice fermions, Phys. Lett. B85 (1979) 100.

[55] G. Bergner, Symmetries an the methods of quantum field theory: Supersymmetry on a space-time lattice, Ph.D. thesis, Friedrich Schiller University of Jena (2009).

[56] M. N. . G. Barkema, Monte Carlo Methods in Statistical Physics, Oxford Uinversity Press, 1999.

[57] D. P. L. . K. Binder, Monte Carlo Simulations in Statistical Physics, Cambridge University Press, 2000.

[58] M. A. Clark and A. D. Kennedy, Accelerating dynamical fermion computations using the rational hybrid Monte Carlo (RHMC) algorithm with multiple pseudofermion fields, Phys. Rev. Lett. 98 (2007) 051601 |arXiv:hep-lat/0608015].

[59] R. Frezzotti and K. Jansen, A polynomial hybrid Monte Carlo algorithm, Phys. Lett. B402 (1997) 328 [arXiv:hep-lat/9702016].

[60] T. Kästner, G. Bergner, S. Uhlmann, A. Wipf and C. Wozar, Two-Dimensional Wess-Zumino Models at Intermediate Couplings, Phys. Rev. D78 (2008) 095001 arXiv:0807.1905.

[61] R. Toral and A. L. Ferreira, Generalized hybrid Monte Carlo, arXiv:hep-lat/9409014

[62] L. O'Raifeartaigh, A. Wipf and H. Yoneyama, The constraint effective potential, Nucl. Phys. B271 (1986) 653.

[63] A. M. Ferrenberg and R. H. Swendsen, New Monte Carlo Technique for Studying Phase Transitions, Phys. Rev. Lett. 61 (1988) 2635.

[64] B. A. Berg and T. Neuhaus, Multicanonical algorithms for first order phase transitions, Phys. Lett. B267 (1991) 249.

[65] E. Gozzi, Functional Integral Approach To Parisi-Wu Stochastic Quantization: Scalar Theory, Phys. Rev. D28 (1983) 1922.

[66] H. Boschi-Filho and C. Farina, Generalized thermal zeta functions, Phys. Lett. A205 (1995) 255 [arXiv:hep-th/9505154].

[67] U. Ellwanger, C. Hugonie and A. M. Teixeira, The Next-to-Minimal Supersymmetric Standard Model, Phys. Rept. 496 (2010) 1 arXiv:0910.1785.

[68] S. Ferrara, Supersymmetric Gauge Theories in Two-Dimensions, Nuovo Cim. Lett. 13 (1975) 629.

[69] A. Schiller and J. Ranft, The (1+1)-dimensional N=2 Wess-Zumino model on the lattice in the local Hamiltonian method, J. Phys. G12 (1986) 935

[70] S. Catterall and S. Karamov, Exact lattice supersymmetry: the two-dimensional N=2 Wess-Zumino model, Phys. Rev. D65 (2002) 094501 arXiv:hep-lat/0108024].

[71] C. Wozar, G. Bergner, T. Kästner, S. Uhlmann and A. Wipf, Numerical Investigation of the 2D N=2 Wess-Zumino Model, PoS LATTICE2008 (2008) 234 arXiv:0809.2176.

[72] J. Bartels and J. B. Bronzan, Supersymmetry on a lattice, Phys. Rev. D28 (1983) 818

[73] J. Ranft and A. Schiller, Hamiltonian monte carlo study of $(1+1)$-dimensional models with restricted supersymmetry on the lattice, Phys. Lett. B138 (1984) 166

[74] M. Beccaria, M. Campostrini and A. Feo, Supersymmetry breaking in two dimensions: The lattice $n=1$ wess-zumino model, Phys. Rev. D69 (2004) 095010 arXiv:hep-lat/0402007.

[75] M. Beccaria, G. F. De Angelis, M. Campostrini and A. Feo, Phase diagram of the lattice Wess-Zumino model from rigorous lower bounds on the energy, Phys. Rev. D70 (2004) 035011 |arXiv:hep-lat/0405016|.

[76] C. Wetterich, Exact evolution equation for the effective potential, Phys. Lett. B301 (1993) 90.

[77] F. Synatschke, H. Gies and A. Wipf, Phase Diagram and Fixed-Point Structure of two dimensional N=1 Wess-Zumino Models, Phys. Rev. D80 (2009) 085007 arXiv:0907.4229.

[78] A. Salam and J. A. Strathdee, On Goldstone Fermions, Phys. Lett. B49 (1974) 465.

[79] N. D. Mermin and H. Wagner, Absence of ferromagnetism or antiferromagnetism in one-dimensional or two-dimensional isotropic Heisenberg models, Phys. Rev. Lett. 17 (1966) 1133.

[80] S. Catterall and S. Karamov, A lattice study of the two-dimensional Wess-Zumino model, Phys. Rev. D68 (2003) 014503 arXiv:hep-lat/0305002].

[81] S.-J. Chang, The Existence of a Second Order Phase Transition in the Two-Dimensional phi**4 Field Theory, Phys. Rev. D13 (1976) 2778.

[82] W. Loinaz and R. S. Willey, Monte Carlo simulation calculation of critical coupling constant for continuum $\phi^{4}(2)$, Phys. Rev. D58 (1998) 076003 arXiv:hep-lat/9712008].

[83] D. Schaich and W. Loinaz, An improved lattice measurement of the critical coupling in $\phi_{2}^{4}$ theory, Phys. Rev. D79 (2009) 056008 arXiv:0902.0045].

[84] K. Binder, Finite size scaling analysis of Ising model block distribution functions, Z. Phys. B43 (1981) 119.

[85] D. Kadoh and H. Suzuki, Supersymmetric nonperturbative formulation of the WZ model in lower dimensions, Phys. Lett. B684 (2010) 167 arXiv:0909.3686 
[86] I. Montvay, Majorana fermions on the lattice, arXiv:hep-lat/0108011

[87] X. Jia and S. Chakravarty, Quantum dynamics of an Ising spin chain in a random transverse field, Phys. Rev. B74 (2006) 172414 arXiv:cond-mat/0607703.

[88] J. Rubow and U. Wolff, A Factorization algorithm to compute Pfaffians, arXiv:1102.3576

[89] S. Catterall and S. Karamov, A two-dimensional lattice model with exact supersymmetry, Nucl. Phys. Proc. Suppl. 106 (2002) 935 arXiv:hep-lat/0110071].

[90] D. Baumgartner and U. Wenger, Simulation of supersymmetric models on the lattice without a sign problem, arXiv:1104.0213

[91] C. Chen, E. Dzienkowski and J. Giedt, Lattice Wess-Zumino model with Ginsparg-Wilson fermions: One-loop results and GPU benchmarks, arXiv: 1005.3276 